\title{
Holomorphic Poisson Manifolds and Holomorphic Lie Algebroids
}

\section{Camille Laurent-Gengoux ${ }^{1}$, Mathieu Stiénon ${ }^{2}$ and Ping $\mathrm{Xu}^{3}$}

${ }^{1}$ Département de mathématiques, Université de Poitiers, 86962 Futuroscope-Chasseneuil, France, ${ }^{2}$ Departement Mathematik, E.T.H. Zürich, 8092 Zürich, Switzerland and ${ }^{3}$ Department of Mathematics, Penn State University, University Park, PA 16802, USA

Correspondence to be sent to: ping@math.psu.edu

Dedicated to the memory of Paulette Libermann

We study holomorphic Poisson manifolds and holomorphic Lie algebroids from the viewpoint of real Poisson geometry. We give a characterization of holomorphic Poisson structures in terms of the Poisson Nijenhuis structures of Magri-Morosi and describe a double complex that computes the holomorphic Poisson cohomology. A holomorphic Lie algebroid structure on a vector bundle $A \rightarrow X$ is shown to be equivalent to a matched pair of complex Lie algebroids $\left(T^{0,1} X, A^{1,0}\right)$, in the sense of Lu. The holomorphic Lie algebroid cohomology of $A$ is isomorphic to the cohomology of the elliptic Lie algebroid $T^{0,1} X \bowtie A^{1,0}$. In the case when $(X, \pi)$ is a holomorphic Poisson manifold and $A=\left(T^{*} X\right)_{\pi}$, such an elliptic Lie algebroid coincides with the Dirac structure corresponding to the associated generalized complex structure of the holomorphic Poisson manifold.

\section{Introduction}

The aim of this paper is to solve several problems naturally arisen in studying the connection between holomorphic Poisson manifolds and holomorphic Lie algebroids with real Poisson geometry.

Received February 14, 2008; Revised June 22, 2008; Accepted June 30, 2008

Communicated by Prof. Michael Finkelberg

(C) The Author 2008. Published by Oxford University Press. All rights reserved. For permissions, please e-mail: journals.permissions@oxfordjournals.org. 
Holomorphic Poisson structures appear naturally in many places $[4,5,12-14,20$, 23, 25, 37, 42]. For instance, any semisimple complex Lie group admits a natural Poisson group structure [10,11,40], which is holomorphic. Its dual is also a holomorphic Poisson group. Indeed, one of the simplest types of examples of holomorphic Poisson manifolds are the Lie-Poisson structures on the dual of complex Lie algebras. Holomorphic Poisson structures were also studied from the point of view of algebraic geometry by Bondal [2] and Polishchuk [39] in the middle of the 90s. Recently, holomorphic Poisson structures were linked to generalized complex geometry [16-19, 24].

There are several equivalent ways of defining holomorphic Poisson structures. One simple definition is, like in the real case, a holomorphic bivector field $\pi$ (i.e. $\pi \in$ $\Gamma\left(\wedge^{2} T^{1,0} X\right)$ such that $\left.\bar{\partial} \pi=0\right)$ satisfying the equation $[\pi, \pi]=0$. Since $\wedge^{2} T_{\mathbb{C}} X=\wedge^{2} T X \oplus$ $i \wedge^{2} T X$, for any $\pi \in \Gamma\left(\wedge^{2} T_{\mathbb{C}} X\right)$, we can write $\pi=\pi_{R}+i \pi_{I}$, where $\pi_{R}$ and $\pi_{I} \in \Gamma\left(\wedge^{2} T X\right)$ are bivector fields on the underlying real manifold $X$.

Problem 1. Are $\pi_{R}$ and $\pi_{I}$ Poisson structures? And conversely, given two Poisson structures $\pi_{R}$ and $\pi_{I}$, when does $\pi=\pi_{R}+i \pi_{I}$ define a holomorphic Poisson structure?

We give an affirmative answer to the first question. As for the second, we show that $\pi=\pi_{R}+i \pi_{I}$ is holomorphic Poisson if and only if $\left(\pi_{I}, J\right)$ is a Poisson Nijenhuis structure and $\pi_{R}^{\sharp}=J \circ \pi_{I}^{\sharp}$. Thus $\left(\pi_{R}, \pi_{I}\right)$ is a bi-Hamiltonian structure on $X$.

Poisson Nijenhuis structures were introduced by Magri and Morosi [34, 35] in their study of bi-Hamiltonian systems, and were intensively studied afterward [27, 43]. A Poisson Nijenhuis structure $[26,27]$ on a manifold $X$ consists of a pair $(\pi, N)$, where $\pi$ is a Poisson tensor on $X$ and $N: T X \rightarrow T X$ is a Nijenhuis tensor that satisfies some compatibility conditions (see Section 2.3 for the precise conditions). By a Nijenhuis tensor, we mean a $(1,1)$-tensor on $X$ with vanishing Nijenhuis torsion.

Since Poisson Nijenhuis structures are related to generalized complex structures [8, 41], as a consequence, we recover the well-known correspondence between holomorphic Poisson structures and generalized complex structures (of a special type) $[17,19]$.

Another natural question is:

Problem 2. Given a holomorphic Poisson structure $\pi=\pi_{R}+i \pi_{I}$, are the holomorphic symplectic foliation of $\pi$ and the symplectic foliations of $\pi_{R}$ and $\pi_{I}$ related? 
Indeed, we show that all these symplectic foliations coincide. Also for a holomorphic symplectic 2-form $\omega=\omega_{R}+i \omega_{I}$, we show that the real and imaginary parts of its holomorphic Poisson tensor are, up to a constant scalar, the Poisson tensors corresponding to $\omega_{R}$ and $\omega_{I}$, respectively.

Lie algebroids are an extremely powerful tool in Poisson geometry. Indeed the Lie algebroid structures on a given vector bundle are in one-one correspondence with the so called fiberwise linear Poisson structures on the dual bundle. This correspondence extends to the holomorphic context; any holomorphic Lie algebroid structure on the vector bundle $A \rightarrow X$ gives rise to a fiberwise linear holomorphic Poisson structure on $A^{*} \rightarrow X$. Thus, the real and imaginary parts of this holomorphic Poisson structure are fiberwise linear Poisson structures on the dual bundle (being considered as a real vector bundle). Hence, one obtains two real Lie algebroid structures $A_{\Re}$ and $A_{\Im}$, respectively.

Problem 3. Obtain an explicit description of the Lie algebroid structures $A_{\Re}$ and $A_{\mathfrak{\Im}}$ in terms of the holomorphic Lie algebroid structure on $A$.

Let $A \rightarrow X$ be a vector bundle endowed with a holomorphic Lie algebroid structure. Extending the Lie bracket on the space of holomorphic sections of $A \rightarrow X$ to the space of all smooth sections so as to preserve the Leibniz rule, we get a real Lie algebroid structure $A_{R}$ on the bundle $A \rightarrow X$. It turns out that, up to a scalar constant, $A_{\Re}$ is isomorphic to $A_{R}$. The multiplication by $\sqrt{-1}$ in the fibers of $A$ defines a real vector bundle map $j: A_{R} \rightarrow A_{R}$ over the identity map satisfying $j^{2}=-$ id.

We prove that the Nijenhuis torsion of $j: A_{R} \rightarrow A_{R}$ vanishes and that, up to a scalar constant, $A_{\Im}$ is isomorphic to $\left(A_{R}\right)_{j}$, the deformation of the Lie algebroid $A_{R}$ by $j$. Extending $j$ by $\mathbb{C}$-linearity, we get a bundle map $j: A_{\mathbb{C}} \rightarrow A_{\mathbb{C}}$ with $j^{2}=-\mathrm{id}$. Since the Nijenhuis torsion of $j$ vanishes, its eigenbundles $A^{1,0}$ and $A^{0,1}$ with eigenvalues $i$ and $-i$ are complex Lie algebroids.

There is yet another connection between Poisson manifolds and Lie algebroids. Given a Poisson manifold $(X, \pi)$, it is well known that $T^{*} X$ carries a natural Lie algebroid structure. This holds for holomorphic Poisson structures as well. Namely, if $(X, \pi)$ is a holomorphic Poisson manifold, then $T^{*} X$ is naturally a holomorphic Lie algebroid, denoted by $\left(T^{*} X\right)_{\pi}$. On the other hand, as highlighted earlier, each holomorphic Poisson structure corresponds to a generalized complex structure $\mathbb{J}$, whose $(-i)$-eigenbundle $L$ is a Dirac structure and thus a complex Lie algebroid [6, 44].

Problem 4. What is the precise relation between the holomorphic Lie algebroid $\left(T^{*} X\right)_{\pi}$ and the complex Lie algebroid $L$ ? 
A key ingredient to answer this problem is the notion of matched pairs studied by Lu, Mackenzie, and Mokri $[31,32,36]$. We show that $\left(T^{0,1} X,\left(T^{1,0} X\right)_{\pi}^{*}\right)$ is a matched pair (here $\left(T^{1,0} X\right)_{\pi}^{*}=A^{1,0}$ for $\left.A=\left(T^{*} X\right)_{\pi}\right)$ and $T^{0,1} X \bowtie\left(T^{1,0} X\right)_{\pi}^{*}$ (see Theorem 4.2 for the definition of $\bowtie$ ) is isomorphic to $L$. Furthermore, we prove that the holomorphic Poisson cohomology of $\pi$, which is defined to be the holomorphic Lie algebroid cohomology of $\left(T^{*} X\right)_{\pi}$, is isomorphic to the cohomology of the elliptic Lie algebroid $T^{0,1} X \bowtie\left(T^{1,0} X\right)_{\pi}^{*}$. This leads to our next problem:

Problem 5. Given an arbitrary holomorphic Lie algebroid $A$, find a complex Lie algebroid $L$ whose cohomology groups are isomorphic to those of $A$.

The cohomology of a holomorphic Lie algebroid $A$ is the cohomology of the complex of sheaves $\left(\Omega_{A^{\bullet}}, d_{A}\right)$, as introduced by Evens-Lu-Weinstein [15] (see Definition 4.10), while the cohomology of a complex (smooth) Lie algebroid $L$ is the cohomology of the cochain complex $\left(\Gamma\left(\wedge^{\bullet} L^{*}\right), d_{L}\right)$. So, in a certain sense, solving the problem above amounts to finding a Dolbeault type of resolution for arbitrary holomorphic Lie algebroids.

The solution is $L=T^{0,1} X \bowtie A^{1,0}$. Indeed, we show that $A$ is holomorphic if and only if $\left(T^{0,1} X, A^{1,0}\right)$ is a matched pair (see Theorem 4.8); one may thus form the complex Lie algebroid $T^{0,1} X \bowtie A^{1,0}$, which is in fact an elliptic Lie algebroid in the sense of Block [1]. The Lie algebroid cohomology of the latter can be expressed as the total cohomology of a double complex.

The following notations are widely used in the sequel. For a manifold $M$, we use $q_{M}$ to denote the projection $T M \rightarrow M$. And given a complex manifold $X, T_{\mathbb{C}} X$ is shorthand for the complexified tangent bundle $T X \otimes \mathbb{C}$ while $T^{1,0} X$ (resp. $T^{0,1} X$ ) stands for the $+i$ (respectively $-i$-) eigenbundle of the almost complex structure. By $\mathfrak{X}^{k, l}(X)$, we denote the space of sections of $\wedge^{k} T^{1,0} X \otimes \wedge^{l} T^{0,1} X \rightarrow X$, and by $\Omega^{k, l}(X)$, the space of differential forms of type $(k, l)$. For a Lie algebroid $A$, the Nijenhuis torsion [26, 27] of a bundle map $\phi: A \rightarrow A$ over the identity is denoted $\mathcal{N}_{\phi}$, which is a section in $\Gamma\left(\wedge^{2} A^{*} \otimes A\right)$ defined by

$$
\mathcal{N}_{\phi}(V, W)=[\phi V, \phi W]-\phi([\phi V, W]+[V, \phi W]-\phi[V, W]), \quad \forall V, W \in \Gamma(A)
$$

When $A$ is the Lie algebroid $T X$ and $\phi: T X \rightarrow T X$ is a $(1,1)$-tensor, the Nijenhuis torsion $\mathcal{N}_{\phi}$ is a $(2,1)$-tensor on $X$.

Note that the modular classes of holomorphic Lie algebroids were studied by Evens-Lu-Weinstein [15] and Huebschmann [21] while the modular classes of holomorphic Poisson manifolds were studied by Brylinski-Zuckerman [5]. In a separate paper, we 
will investigate the relation between these modular classes and their counterparts in real Poisson geometry, and in particular with the modular classes of Poisson Nijenhuis manifolds recently studied by Damianou-Fernandes [9] and Kosmann-Schwarzbach-Magri [28].

\section{Holomorphic Poisson manifolds}

\subsection{Definition}

Definition 2.1. A holomorphic Poisson manifold is a complex manifold $X$ whose sheaf of holomorphic functions $\mathcal{O}_{X}$ is a sheaf of Poisson algebras.

By a sheaf of Poisson algebras over $X$, we mean that, for each open subset $U \subset X$, the ring $\mathcal{O}_{X}(U)$ is endowed with a Poisson bracket such that all restriction maps $\mathcal{O}_{X}(U) \rightarrow \mathcal{O}_{X}(V)$ (for arbitrary open subsets $\left.V \subset U \subset X\right)$ are morphisms of Poisson algebras. Moreover, given an open subset $U \subset X$, an open covering $\left\{U_{i}\right\}_{i \in I}$ of $U$, and a pair of functions $f, g \in \mathcal{O}_{X}(U)$, then the local data $\left\{\left.f\right|_{U_{i}},\left.g\right|_{U_{i}}\right\}(i \in I)$ glue up to $\left\{\left.f\right|_{U},\left.g\right|_{U}\right\}$ if they coincide on the overlaps $U_{i} \cap U_{j}$.

Lemma 2.2. On a given complex manifold $X$, holomorphic Poisson structures are in one-to-one correspondence with holomorphic bivector fields $\pi$ (i.e. $\pi \in \Gamma\left(\wedge^{2} T^{1,0} X\right)$ such that $\bar{\partial} \pi=0)$, satisfying the equation $[\pi, \pi]=0$.

Proof. This is a standard result. For completeness, let us sketch a proof here. Choose any complex coordinate chart $(U, \phi)$, which identifies $U \subset X$ with an open ball of $\mathbb{C}^{n}$. As in the smooth case, $\mathcal{O}_{X}(U)$ is a Poisson algebra that is equivalent to the existence of a holomorphic bivector field $\pi_{U}$ on $U$, satisfying the relation $\left[\pi_{U}, \pi_{U}\right]=0$. Moreover, the compatibility condition on the restriction maps implies that there indeed exists a holomorphic bivector field $\pi$ on $X$, whose restriction to $U$ is $\pi_{U}$ for all such open subsets $U$.

\subsection{Associated real Poisson structures}

Since $\wedge^{2} T_{\mathbb{C}} X=\wedge^{2} T X \oplus i \wedge^{2} T X$, for any $\pi \in \Gamma\left(\wedge^{2} T_{\mathbb{C}} X\right)$, we can write $\pi=\pi_{R}+i \pi_{I}$, where $\pi_{R}$ and $\pi_{I} \in \Gamma\left(\wedge^{2} T X\right)$ are (real) bivector fields on $X$ (seen as a real manifold by forgetting the complex structure). Note that sections of $\wedge^{2} T_{\mathbb{C}} X$ (in particular of $\wedge^{2} T^{1,0} X$ ) can be seen 
as bidifferential operators on $C^{\infty}(M, \mathbb{C})$. The real bivector fields $\pi_{R}$ and $\pi_{I}$ are then the real and imaginary parts of these bidifferential operators.

Both $\pi_{R}$ and $\pi_{I}$ define brackets $\{\cdot, \cdot\}_{R}$ and $\{\cdot, \cdot\}_{I}$ on $C^{\infty}(M, \mathbb{R})$ in the standard way. These extend to $C^{\infty}(M, \mathbb{C})$ by $\mathbb{C}$-linearity. The next lemma describes such an extension.

\section{Lemma 2.3.}

(a) Under the direct sum decomposition

$$
\wedge^{2} T_{\mathbb{C}} X=\wedge^{2} T^{1,0} X \oplus\left(T^{1,0} X \wedge T^{0,1} X\right) \oplus \wedge^{2} T^{0,1} X
$$

we have

$$
\pi_{R}=\frac{\pi}{2}+0+\frac{\bar{\pi}}{2} \quad \text { and } \quad \pi_{I}=\frac{\pi}{2 i}+0+\frac{-\bar{\pi}}{2 i} .
$$

(b) $\forall f, g \in \mathcal{O}_{X}(U)$, we have the following relations:

$$
\begin{array}{lll}
\{f, g\}_{R}=\frac{1}{2}\{f, g\}, & \{\bar{f}, \bar{g}\}_{R}=\frac{1}{2} \overline{\{f, g\}}, & \{f, \bar{g}\}_{R}=0 \\
\{f, g\}_{I}=-\frac{i}{2}\{f, g\}, & \{\bar{f}, \bar{g}\}_{I}=\frac{i}{2} \overline{\{f, g\},} \quad\{f, \bar{g}\}_{I}=0 .
\end{array}
$$

(c) Both $\pi_{R}$ and $\pi_{I}$ are Poisson tensors.

Proof. (a) Immediate. (b) If $f, g \in \mathcal{O}_{X}(U)$, then $\bar{f}$ and $\bar{g}$ are antiholomorphic. Hence $\partial \bar{f}=0=\partial \bar{g}$. Therefore, $\pi(\partial f, \partial g)=\{f, g\}, \pi(\partial \bar{f}, \partial g)=0$ and $\pi(\partial \bar{f}, \partial \bar{g})=0$. The conclusion follows. (c) It suffices to prove the Jacobi identity for $\{\cdot, \cdot\}_{R}$ and $\{\cdot, \cdot\}_{I}$. From (b), it follows that it holds for holomorphic functions in $\mathcal{O}_{X}(U)$. It follows from the Leibniz rule that the Jacobi identity holds for all complex-valued smooth functions in $C^{\infty}(X, \mathbb{C})$. This concludes the proof.

As an immediate consequence, we have the following

Corollary 2.4. For all $f, g \in \mathcal{O}_{X}(U)$, we have

$$
\begin{array}{ll}
\{\Re f, \Re g\}_{R}=\frac{1}{4} \Re\{f, g\}, & \{\Re f, \Re g\}_{I}=\frac{1}{4} \Im\{f, g\}, \\
\{\mathfrak{\Im} f, \Im g\}_{R}=-\frac{1}{4} \Re\{f, g\}, & \{\Im f, \Im g\}_{I}=-\frac{1}{4} \Im\{f, g\}, \\
\{\Re f, \Im g\}_{R}=\frac{1}{4} \Im\{f, g\}, & \{\Re f, \Im g\}_{I}=-\frac{1}{4} \Re\{f, g\} .
\end{array}
$$

where $\Re f$ and $\Im f$ stand for the real and imaginary part of the function $f$, respectively. 
Thus, in a local chart $\left(z_{1}=x_{1}+i y_{1}, \ldots, z_{n}=x_{n}+i y_{n}\right)$ of complex coordinates of $X$, we have

$$
\begin{array}{ll}
\left\{x_{i}, x_{j}\right\}_{R}=\frac{1}{4} \Re\left\{z_{i}, z_{j}\right\}, & \left\{x_{i}, x_{j}\right\}_{I}=\frac{1}{4} \Im\left\{z_{i}, z_{j}\right\}, \\
\left\{y_{i}, y_{j}\right\}_{R}=-\frac{1}{4} \Re\left\{z_{i}, z_{j}\right\}, & \left\{y_{i}, y_{j}\right\}_{I}=-\frac{1}{4} \Im\left\{z_{i}, z_{j}\right\}, \\
\left\{x_{i}, y_{j}\right\}_{R}=\frac{1}{4} \Im\left\{z_{i}, z_{j}\right\}, & \left\{x_{i}, y_{j}\right\}_{I}=-\frac{1}{4} \Re\left\{z_{i}, z_{j}\right\} .
\end{array}
$$

\subsection{Poisson Nijenhuis structures}

Lemma 2.5. Let $\pi=\pi_{R}+i \pi_{I} \in \Gamma\left(\wedge^{2} T_{\mathbb{C}} X\right)$ with $\pi_{R}, \pi_{I} \in \Gamma\left(\wedge^{2} T X\right)$. Then, $\pi \in \Gamma\left(\wedge^{2} T^{1,0} X\right)$ iff $\pi_{R}^{\sharp}=\pi_{I}^{\sharp} \circ J^{*}$, where $J: T X \rightarrow T X$ is the almost complex structure on $X$.

Proof. We have

$$
\begin{array}{ll} 
& \pi \in \Gamma\left(\wedge^{2} T^{1,0} X\right) \\
\Leftrightarrow & \pi(\alpha, \beta)=0, \forall \alpha \in \Omega^{0,1}(X), \beta \in \Omega_{\mathbb{C}}^{1}(X) \\
\Leftrightarrow & \pi(\alpha, \beta)=0, \alpha=\frac{1+i J^{*}}{2}\left(\alpha^{\prime}\right), \forall \alpha^{\prime}, \beta \in \Omega_{\mathbb{C}}^{1}(X) \\
\Leftrightarrow & \pi^{\sharp} \circ\left(\frac{1+i J^{*}}{2}\right)=0 \\
\Leftrightarrow & i \pi^{\sharp}=\pi^{\sharp} \circ J^{*} \\
\Leftrightarrow & 2 \pi_{R}^{\sharp} \circ J^{*}=\left(\pi^{\sharp}+\bar{\pi}^{\sharp}\right) \circ J^{*}=i\left(\pi^{\sharp}-\bar{\pi}^{\sharp}\right)=-2 \pi_{I}^{\sharp} \\
\Leftrightarrow & \pi_{R}^{\sharp}=\pi_{I}^{\sharp} \circ J^{*}
\end{array}
$$

Recall that a Poisson Nijenhuis structure $[26,27]$ on a manifold $X$ consists of a pair $(\pi, N)$, where $\pi$ is a Poisson tensor on $X$ and $N: T X \rightarrow T X$ is a Nijenhuis tensor such that the following compatibility conditions are satisfied:

$$
\begin{gathered}
N \circ \pi^{\sharp}=\pi^{\sharp} \circ N^{*} \\
{[\alpha, \beta]_{\pi_{N}}=\left[N^{*} \alpha, \beta\right]_{\pi}+\left[\alpha, N^{*} \beta\right]_{\pi}-N^{*}[\alpha, \beta]_{\pi},}
\end{gathered}
$$

where $\pi_{N}$ is the bivector field on $X$ defined by the relation $\pi_{N}^{\sharp}=\pi^{\sharp} \circ N^{*}$, and for any bivector field $\hat{\pi}$ on $M$,

$$
[\alpha, \beta]_{\hat{\pi}}:=\mathcal{L}_{\hat{\pi}^{\sharp} \alpha}(\beta)-\mathcal{L}_{\hat{\pi}^{\sharp} \beta}(\alpha)-d(\hat{\pi}(\alpha, \beta)), \quad \forall \alpha, \beta \in \Omega^{1}(M) .
$$


Proposition 2.6. Let $X$ be a complex manifold with associated almost complex structure $J$. Then, $\pi=\pi_{R}+i \pi_{I}$, where $\pi_{R}, \pi_{I} \in \Gamma\left(\wedge^{2} T X\right)$ is a holomorphic Poisson structure on $X$ iff the pair $\left(\pi_{I}, J\right)$ is a Poisson Nijenhuis structure and $\pi_{R}^{\sharp}=\pi_{I}^{\sharp} \circ J^{*}$.

Proof. First, observe that, for all $f \in C^{\infty}(X, \mathbb{C})$ and $\alpha, \beta \in \Omega_{\mathbb{C}}^{1}(X)$, one has

$$
[\alpha, f \beta]_{\pi_{R}}=\left(\pi_{R}^{\sharp} \alpha\right)(f) \beta+f[\alpha, \beta]_{\pi_{R}}
$$

and

$$
\begin{aligned}
& {\left[J^{*} \alpha, f \beta\right]_{\pi_{I}}+\left[\alpha, J^{*} f \beta\right]_{\pi_{I}}-J^{*}[\alpha, f \beta]_{\pi_{I}}} \\
& \quad=\left(\pi_{I}^{\sharp} J^{*} \alpha\right)(f) \beta+f\left(\left[J^{*} \alpha, \beta\right]_{\pi_{I}}+\left[\alpha, J^{*} \beta\right]_{\pi_{I}}-J^{*}[\alpha, \beta]_{\pi_{I}}\right) .
\end{aligned}
$$

Therefore, since by Lemma 2.5, we have $\pi_{R}^{\sharp}=\pi_{I}^{\sharp} \circ J^{*}$, it suffices to check the compatibility condition 2 for $\alpha=d f$ or $d \bar{f}$ and $\beta=d g$ or $d \bar{g}$ with $f$ and $g \in \mathcal{O}_{X}(U)$.

An easy but cumbersome computation, making use of the relations of Lemma 2.3 and the well-known equivalences

$$
f \in \mathcal{O}_{X}(U) \quad \Longleftrightarrow \quad J^{*} d f=i d f \quad \Longleftrightarrow \quad J^{*} d \bar{f}=-i d \bar{f},
$$

shows that the Poisson Nijenhuis compatibility of $\pi_{I}$ and $J$ is equivalent to the closure of $\mathcal{O}_{X}(U)$ under the Poisson bracket of functions associated to $\pi$.

For example, $\forall f, g \in \mathcal{O}_{X}(U)$ :

$$
\begin{aligned}
& {\left[J^{*} d f, d g\right]_{\pi_{I}}+\left[d f, J^{*} d g\right]_{\pi_{I}}-J^{*}[d f, d g]_{\pi_{I}}-[d f, d g]_{\pi_{R}}} \\
& \quad=[i d f, d g]_{\pi_{I}}+[d f, i d g]_{\pi_{I}}-J^{*} d\{f, g\}_{I}-d\{f, g\}_{R} \\
& \quad=2 i d\{f, g\}_{I}-J^{*} d\{f, g\}_{I}-d\{f, g\}_{R} \\
& \quad=d\{f, g\}+\frac{i}{2} J^{*} d\{f, g\}-\frac{1}{2} d\{f, g\} \\
& \quad=\frac{i}{2}\left(J^{*} d\{f, g\}-i d\{f, g\}\right) .
\end{aligned}
$$

Theorem 2.7. Given a complex manifold $X$ with associated almost complex structure $J$, the following are equivalent:

(a) $\pi=\pi_{R}+i \pi_{I} \in \Gamma\left(\wedge^{2} T^{1,0} X\right)$ is a holomorphic Poisson bivector field;

(b) $\left(\pi_{I}, J\right)$ is a Poisson Nijenhuis structure on $X$ and $\pi_{R}^{\sharp}=\pi_{I}^{\sharp} \circ J^{*}$; 
(c) the endomorphism

$$
\mathbb{J}_{\pi}=\left(\begin{array}{cc}
J & \pi_{I}^{\sharp} \\
0 & -J^{*}
\end{array}\right)
$$

of $T M \oplus T^{*} M$ is a generalized complex structure and $\pi_{R}^{\sharp}=\pi_{I}^{\sharp} \circ J^{*}$.

Proof. (a) $\Longleftrightarrow$ (b) This is Proposition 2.6. (b) $\Longleftrightarrow$ (c) The equivalence follows from [41, Theorem 7.6] (see also [8]).

Remark 2.8. It is well known that a holomorphic Poisson structure gives rise to a generalized complex structure. The holomorphic Poisson tensor is a strong Hamiltonian operator in the sense of Liu-Weinstein-Xu [29], which deforms the Dirac structure on $T_{\mathbb{C}} X \oplus T_{\mathbb{C}}^{*} X$ associated to the usual complex structure seen as a generalized complex structure $[1,17,24]$.

Remark 2.9. The equivalence of (a) and (b) in Theorem 2.7 was also known to Lu [30].

\subsection{Holomorphic symplectic manifolds}

Let $(X, \omega)$ be a holomorphic symplectic manifold, where $\omega \in \Omega^{2,0}(X)$ is the holomorphic symplectic 2-form whose corresponding holomorphic Poisson bivector field is denoted by $\pi=\pi_{R}+i \pi_{I} \in \Gamma\left(\wedge^{2} T^{1,0} X\right)$. Let $\omega_{R}, \omega_{I} \in \Omega^{2}(X)$ be the real and imaginary parts of $\omega_{\text {, }}$ i.e. $\omega=\omega_{R}+i \omega_{I}$. By the holomorphic Darboux theorem, both $\omega_{R}$ and $\omega_{I}$ are symplectic 2-forms.

Proposition 2.10. The Poisson bivector fields corresponding to $\omega_{R}$ and $\omega_{I}$ are $4 \pi_{R}$ and $-4 \pi_{I}$, respectively.

Proof. The holomorphic Darboux theorem asserts that, in a neighborhood of each point, there exist complex symplectic coordinates $\left(z_{1}, \ldots, z_{n}, z_{1}^{\prime}, \ldots, z_{n}^{\prime}\right)$ so that $\omega$ can be written as

$$
\omega=\sum_{k=1}^{n} d z_{k} \wedge d z_{k}^{\prime}
$$


In terms of real coordinates, defined by

$$
z_{k}=x_{k}+i y_{k}, \quad z_{k}^{\prime}=x_{k}^{\prime}+i y_{k}^{\prime}
$$

for $k=1, \ldots, n$, we have

$$
\left\{\begin{array}{l}
\omega_{R}=\sum_{k=1}^{n}\left(d x_{k} \wedge d x_{k}^{\prime}-d y_{k} \wedge d y_{k}^{\prime}\right) \\
\omega_{I}=\sum_{k=1}^{n}\left(d x_{k} \wedge d y_{k}^{\prime}+d y_{k} \wedge d x_{k}^{\prime}\right) .
\end{array}\right.
$$

By $\omega_{R}^{-1}$ and $\omega_{I}^{-1}$, we denote the Poisson bivector fields corresponding to $\omega_{R}$ and $\omega_{I}$, respectively. Thus, we have

$$
\left\{\begin{aligned}
\pi & =-\sum_{k=1}^{n} \frac{\partial}{\partial z_{k}} \wedge \frac{\partial}{\partial z_{k}^{\prime}} \\
\omega_{R}^{-1} & =-\sum_{k=1}^{n}\left(\frac{\partial}{\partial x_{k}} \wedge \frac{\partial}{\partial x_{k}^{\prime}}-\frac{\partial}{\partial y_{k}} \wedge \frac{\partial}{\partial y_{k}^{\prime}}\right) \\
\omega_{I}^{-1} & =-\sum_{k=1}^{n}\left(\frac{\partial}{\partial x_{k}} \wedge \frac{\partial}{\partial y_{k}^{\prime}}+\frac{\partial}{\partial y_{k}} \wedge \frac{\partial}{\partial x_{k}^{\prime}}\right) .
\end{aligned}\right.
$$

On the other hand, using the relations $\frac{\partial}{\partial z_{k}}=\frac{1}{2}\left(\frac{\partial}{\partial x_{k}}-i \frac{\partial}{\partial y_{k}}\right)$ and $\frac{\partial}{\partial z_{k}^{\prime}}=\frac{1}{2}\left(\frac{\partial}{\partial x_{k}^{\prime}}-i \frac{\partial}{\partial y_{k}^{\prime}}\right)$, it is simple to see that the real and imaginary parts of $\pi$ are given by

$$
\left\{\begin{array}{l}
\pi_{R}=-\frac{1}{4} \sum_{k=1}^{n}\left(\frac{\partial}{\partial x_{k}} \wedge \frac{\partial}{\partial x_{k}^{\prime}}-\frac{\partial}{\partial y_{k}} \wedge \frac{\partial}{\partial y_{k}^{\prime}}\right), \\
\pi_{I}=\frac{1}{4} \sum_{k=1}^{n}\left(\frac{\partial}{\partial x_{k}} \wedge \frac{\partial}{\partial y_{k}^{\prime}}+\frac{\partial}{\partial y_{k}} \wedge \frac{\partial}{\partial x_{k}^{\prime}}\right) .
\end{array}\right.
$$

The conclusion thus follows immediately. 


\subsection{Symplectic foliation}

Proposition 2.11. Let $(X, \pi)$ be a holomorphic Poisson manifold, and $\pi_{R}$ and $\pi_{I}$ the real and imaginary parts of $\pi$. Then, the symplectic foliations of $\pi_{R}$ and $\pi_{I}$ coincide, and their leaves are exactly the holomorphic symplectic leaves of $\pi$.

Proof. The relation $\pi_{R}^{\sharp}=\pi_{I}^{\sharp} \circ J^{*}$ implies that $\pi_{R}$ and $\pi_{I}$ have the same symplectic leaves, for the distributions $\pi_{R}^{\sharp}\left(T^{*} X\right)$ and $\pi_{I}^{\sharp}\left(T^{*} X\right)$ coincide.

The relation $\pi=\pi_{R}+i \pi_{I}=\pi_{R}+i J \pi_{R}$ implies that, for all $\alpha \in\left(T^{0,1} X\right)^{*}$,

$$
\pi^{\sharp}(\alpha)=(\mathrm{id}+i J) \pi_{R}^{\sharp}(\alpha) .
$$

Since $\alpha=\Re(\alpha)+i J^{*} \Re(\alpha)$, we obtain

$$
\pi^{\sharp}(\alpha)=2(\mathrm{id}+i J) \pi_{R}^{\sharp}(\Re(\alpha)) .
$$

Taking the real part, we obtain

$$
\Re\left(\pi^{\sharp}(\alpha)\right)=2 \pi_{R}^{\sharp}(\Re(\alpha)) .
$$

In particular, the map $T^{0,1} X \rightarrow T X$ sending an element to its real part is an isomorphism from the distribution $\pi^{\sharp}\left(\left(T^{0,1} X\right)^{*}\right)$ to the distribution $\pi_{R}^{\sharp}\left(T^{*} X\right)$, so that the leaves associated to these distributions coincide.

\section{Holomorphic Lie Algebroids}

\subsection{Definition}

Holomorphic Lie algebroids were studied for various purposes in the literature. See $[3,6,15,21,44]$ and references cited there for details. Let us recall its definition below.

The tangent bundle $T X \rightarrow X$ of a complex manifold $X$ is naturally a holomorphic vector bundle. We will denote its sheaf of holomorphic sections, i.e. the sheaf of holomorphic vector fields, by $\Theta_{X}$.

Given a holomorphic vector bundle $A \stackrel{p}{\rightarrow} X$, the sheaf of holomorphic sections $\mathcal{A}$ of $A \rightarrow X$ is the contravariant functor that associates to an open subset $U$ of $X$ the space $\mathcal{A}(U)$ of holomorphic sections of $A \rightarrow X$ over $U$. Similarly, the sheaf of smooth sections $\mathcal{A}_{\infty}$ is the contravariant functor $U \rightarrow \Gamma\left(A_{U}\right)$. Clearly, $\mathcal{A}$ is a sheaf of $\mathcal{O}_{X}$-modules while $\mathcal{A}_{\infty}$ 
is a sheaf of modules where $C_{X}^{\infty}$ denotes the sheaf of complex-valued smooth functions on $X$. Moreover, $\mathcal{A}$ is a subsheaf of $\mathcal{A}_{\infty}$.

Definition 3.1. A holomorphic Lie algebroid is a holomorphic vector bundle $A \rightarrow X$, equipped with a holomorphic bundle map $A \stackrel{\rho}{\rightarrow} T X$, called the anchor map, and a structure of sheaf of complex Lie algebras on $\mathcal{A}$, such that

(a) the anchor map $\rho$ induces a homomorphism of sheaves of complex Lie algebras from $\mathcal{A}$ to $\Theta_{X}$;

(b) and the Leibniz identity

$$
[V, f W]=(\rho(V) f) W+f[V, W]
$$

holds for all $V, W \in \mathcal{A}(U), f \in \mathcal{O}_{X}(U)$ and all open subsets $U$ of $X$.

Remark 3.2. Note that in the definition above, the last axiom implies that the anchor map $\rho$ is automatically a holomorphic bundle map once we assume that it is a complex bundle map.

\subsection{Underlying real Lie algebroid}

By forgetting the complex structure, a holomorphic vector bundle $A \rightarrow X$ becomes a real (smooth) vector bundle, and a holomorphic vector bundle map $\rho: A \rightarrow T X$ becomes a real (smooth) vector bundle map.

Let $A \rightarrow X$ be a holomorphic vector bundle whose underlying real vector bundle is endowed with a Lie algebroid structure $(A, \rho,[\cdot, \cdot])$ such that, for any open subset $U \subset X$,

1. $[\mathcal{A}(U), \mathcal{A}(U)] \subset \mathcal{A}(U)$

2. and the restriction of the Lie bracket $[\cdot, \cdot]$ to $\mathcal{A}(U)$ is $\mathbb{C}$-linear.

Then, the restriction of $[\cdot, \cdot]$ and $\rho$ from $\Gamma(A)$ to $\mathcal{A}$ makes $A$ a holomorphic Lie algebroid.

The following proposition states that any holomorphic Lie algebroid can be obtained out of such a real Lie algebroid, in a unique way.

Proposition 3.3. Given a structure of holomorphic Lie algebroid on the holomorphic vector bundle $A \rightarrow X$ with anchor map $A \stackrel{\rho}{\rightarrow} T X$, there exists a unique structure of real smooth Lie algebroid on the vector bundle $A \rightarrow X$ with respect to the same anchor map $\rho$ such that the inclusion of sheaves $\mathcal{A} \subset \mathcal{A}_{\infty}$ is a morphism of sheaves of real Lie algebras. 
Proof. (i) Unicity. We first prove the unicity. Assume there exist two such Lie algebroid structures on the vector bundle $A \rightarrow X$. The two anchor maps would be equal. And for each open subset $U$ of $X$, the two brackets would coincide on the subspace $\mathcal{A}(U)$ of $\Gamma\left(A_{U}\right)$. Thus, the two brackets would also coincide on the $C^{\infty}(U, \mathbb{R})$-span of $\mathcal{A}(U)$ inside $\Gamma\left(A_{U}\right)$. But for all trivializing open subsets $U$ of $X, \mathcal{A}(U)$ generates $\Gamma\left(A_{U}\right)$. Hence, the two Lie algebroid structures must coincide.

(ii) Existence. We first prove the existence of such a structure of real Lie algebroid. Denote by $j: A \rightarrow A$ the bundle map defining the fiberwise complex structure on $A$.

Recall that, given a Lie algebra $\mathfrak{h}$ and a commutative algebra $F$ over a field $k$ together with a Lie algebra homomorphism $\rho: \mathfrak{h} \rightarrow \operatorname{Der}(F)$, the tensor product $F \otimes_{k} \mathfrak{h}$ is endowed with a natural Lie algebra structure over the field $k$ given by

$$
[f \otimes a, g \otimes b]=f g \otimes[a, b]+f \rho(a) g \otimes b-g \rho(b) f \otimes a,
$$

for all $a, b \in \mathfrak{h}, f, g \in F$. Choose an open subset $U$ of $X$. Applying the previous general fact to the Lie algebra $\mathcal{A}(U)$, the commutative algebra $C^{\infty}(U, \mathbb{C})$ and the anchor map $\rho: \mathcal{A}(U) \rightarrow \operatorname{Der}\left(C^{\infty}(U, \mathbb{C})\right)$, one obtains a Lie algebra structure on $C^{\infty}(U, \mathbb{C}) \otimes_{\mathbb{R}} \mathcal{A}(U)$. Note that this is a real Lie algebra, since $\mathcal{A}(U) \rightarrow \operatorname{Der}\left(C^{\infty}(U, \mathbb{C})\right)$ is $\mathbb{R}$-linear but not $\mathbb{C}$-linear.

Now, for any holomorphic function $h \in \mathcal{O}_{X}(U)$, it follows from Eq. (4) and the Leibniz identity for the holomorphic Lie algebroid $A \rightarrow X$ that

$$
\begin{aligned}
{[f \otimes a, g h \otimes b-g \otimes h b]=} & f g h \otimes[a, b]+f g \rho(a) h \otimes b+f(\rho(a) g) h \otimes b \\
& -f g \otimes h[a, b]-f g \otimes(\rho(a) h) b-f(\rho(a) g) \otimes h b .
\end{aligned}
$$

In other words, the elements of type $f h \otimes a-f \otimes h a$, with $f \in C^{\infty}(U, \mathbb{C}), a \in$ $\mathcal{A}(U)$, and $h \in \mathcal{O}_{X}(U)$, generate an ideal of the Lie algebra $C^{\infty}(U, \mathbb{C}) \otimes_{\mathbb{R}} \mathcal{A}(U)$. As a consequence, the Lie bracket given by equation (4) induces a Lie algebra structure (over $\mathbb{R}$ ) on the quotient $C^{\infty}(U, \mathbb{C}) \otimes_{\mathcal{O}_{X}(U)} \mathcal{A}(U)$ of $C^{\infty}(U, \mathbb{C}) \otimes_{\mathbb{R}} \mathcal{A}(U)$ by the aforementioned ideal.

There is a natural map $C^{\infty}(U, \mathbb{C}) \otimes_{\mathcal{O}_{X}(U)} \mathcal{A}(U) \hookrightarrow \Gamma\left(A_{U}\right)$ mapping $f \otimes a$ to $\Re(f) a+$ $\Im(f) j(a)$, for all $f \in C^{\infty}(U, \mathbb{C})$ and $a \in \mathcal{A}(U)$. This is actually an isomorphism if the open subset $U$ of $X$ is trivializing the holomorphic bundle $\left.A\right|_{U} \rightarrow U$. Indeed, any smooth section of $A \rightarrow X$ over $U$ can be written as a linear combination $\sum_{k=1}^{m} f_{k} a_{k}$, where $a_{k} \in \mathcal{A}(U)$ and $f_{k}$ is a smooth $\mathbb{C}$-valued function on $U$. Therefore, $\Gamma\left(A_{U}\right)$ is a Lie algebra. By construction, the previous Lie bracket restricts to a $\mathbb{C}$-linear bracket on $\mathcal{A}(U)$ and is a Lie-Rinehart algebra over $C^{\infty}(U)$. Hence, $\left.A\right|_{U}$ is a smooth Lie algebroid. By the unicity in (i), one 
obtains a smooth Lie algebroid $A \rightarrow X$ by gluing $\left.A\right|_{U}$ together using an open covering $\left\{U_{i}\right\}$ of $X$.

In the sequel, we will use $A_{R}$ to denote the underlying real Lie algebroid of a holomorphic Lie algebroid $A$. When referring to holomorphic Lie algebroids, we either use Definition 3.1, or the equivalent one, as in Proposition 3.3. In particular, by saying that a real Lie algebroid is a holomorphic Lie algebroid, we mean that it is a holomorphic vector bundle and its Lie bracket on smooth section restricts to a $\mathbb{C}$-linear bracket on $\mathcal{A}(U)$, for all open subsets $U \subset X$.

\subsection{Underlying imaginary Lie algebroid}

Assume that $(A \rightarrow X, \rho,[\cdot, \cdot])$ is a holomorphic Lie algebroid. Consider the bundle map $j: A \rightarrow A$ defining the fiberwise complex structure on $A$. We compute the Nijenhuis torsion of $j$ by considering $A$ as a real Lie algebroid $A_{R}$.

Proposition 3.4. Let $(A \rightarrow X, \rho,[\cdot, \cdot])$ be a holomorphic Lie algebroid and $j: A_{R} \rightarrow A_{R}$ its associated endomorphism. Then, the Nijenhuis torsion of $j$ vanishes.

Proof. Since $T(j)$ is a section in $\Gamma\left(\wedge^{2} A_{R}^{*} \otimes A_{R}\right)$, it suffices to prove that $T(j)(V, W)=0$ for any holomorphic sections $V, W \in \mathcal{A}(U)$, where $U \subset X$ is any open subset. This, however, follows immediately from the $\mathbb{C}$-linearity of $[\cdot, \cdot]$ on $\mathcal{A}(U)$ :

$$
[V, j W]=j[V, W]=[j V, W], \quad \forall V, W \in \mathcal{A}(U) .
$$

Since the Nijenhuis torsion of $j: A_{R} \rightarrow A_{R}$ vanishes, one can define a new (real) Lie algebroid structure on $A$, denoted by $\left(A \rightarrow X, \rho_{j},[\cdot, \cdot]_{j}\right)$, where the anchor $\rho_{j}$ is $\rho \circ j$ and the bracket on $\Gamma(A)$ is given by [26]

$$
[V, W]_{j}=[j V, W]+[V, j W]-j[V, W], \quad \forall V, W \in \Gamma(A) .
$$

In the sequel, $\left(A \rightarrow X, \rho_{j},[\cdot, \cdot]_{j}\right)$ will be called the underlying imaginary Lie algebroid and denoted by $A_{I}$. It is known that

$$
j: A_{I} \rightarrow A_{R}
$$

is a Lie algebroid isomorphism [26]. 


\subsection{Associated complex Lie algebroids}

Let $(A \rightarrow X,[\cdot, \cdot], \rho)$ be a holomorphic Lie algebroid. Complexifying its underlying real Lie algebroid (which was described in Proposition 3.3) by extending the anchor map and the Lie bracket $\mathbb{C}$-linearly, we obtain a complex Lie algebroid:

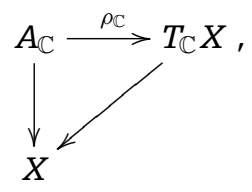

where $A_{\mathbb{C}}=A \otimes \mathbb{C}$. Extending $\mathbb{C}$-linearly the bundle map $j: A \rightarrow A$, we obtain a map of complex vector bundles $j: A_{\mathbb{C}} \rightarrow A_{\mathbb{C}}$ such that $j^{2}=-$ id. Let $A^{1,0} \rightarrow X$ and $A^{0,1} \rightarrow X$ be its eigenbundles with eigenvalues $i$ and $-i$, respectively. It follows from Proposition 3.4 that $\Gamma\left(A^{1,0}\right)$ and $\Gamma\left(A^{0,1}\right)$ are Lie subalgebras of $\Gamma\left(A_{\mathbb{C}}\right)$. Hence, $A^{1,0}$ and $A^{0,1}$ are complex Lie subalgebroids of $A_{\mathbb{C}}$. Note that the map

$$
A \rightarrow A^{1,0}: a \mapsto \frac{1}{2}(a-i j(a))
$$

is an isomorphism of complex vector bundles. Hence, by pulling back all the structures, one obtains a complex Lie algebroid structure on the same complex vector bundle $A \rightarrow$ $X$. Similarly, $a \rightarrow \frac{1}{2}(a+i j(a))$ is an isomorphism of complex vector bundles $A \rightarrow A^{0,1}$. Hence, $A \rightarrow X$ admits another complex Lie algebroid structure. The following proposition describes these complex Lie algebroids explicitly. Its proof is a simple computation and is left to the reader.

Proposition 3.5. Given a holomorphic Lie algebroid $(A \rightarrow X,[\cdot, \cdot], \rho)$, let $(A \rightarrow$ $\left.X,[\cdot, \cdot]_{1,0}, \rho_{1,0}\right)$ and $\left(A \rightarrow X,[\cdot, \cdot]_{0,1}, \rho_{0,1}\right)$ be its associated complex Lie algebroids corresponding to $A^{1,0}$ and $A^{0,1}$, respectively. Then,

$$
\begin{array}{ll}
\rho_{1,0}=\frac{1}{2}\left(\rho+i \rho_{j}\right), & {[\cdot, \cdot]_{1,0}=\frac{1}{2}\left([\cdot, \cdot]+j[\cdot, \cdot]_{j}\right)} \\
\rho_{0,1}=\frac{1}{2}\left(\rho-i \rho_{j}\right), & {[\cdot, \cdot]_{0,1}=\frac{1}{2}\left([\cdot, \cdot]-j[\cdot, \cdot]_{j}\right) .}
\end{array}
$$




\subsection{Cotangent bundle Lie algebroids of holomorphic Poisson manifolds}

In this section, as an example we consider the cotangent bundle Lie algebroid of a holomorphic Poisson manifold and identify various Lie algebroids associated to it, which will be used later on.

Assume that $(X, \pi)$ is a holomorphic Poisson manifold, where $\pi=\pi_{R}+i \pi_{I} \in$ $\Gamma\left(\wedge^{2} T^{1,0} X\right)$. Let $A=\left(T^{*} X\right)_{\pi}$ be its corresponding holomorphic Lie algebroid, which can be defined in a similar way as in the smooth case. To be more precise, let $\Phi$ and $\Psi$, respectively, be the holomorphic bundle maps

$$
\Phi: T X \rightarrow T^{1,0} X, \Phi=\frac{1}{2}(1-i J)
$$

and

$$
\Psi: T^{*} X \rightarrow\left(T^{1,0} X\right)^{*}, \Psi=1-i J^{*},
$$

where $J$ is the almost complex structure on $X$. Define the anchor $\rho:\left(T^{*} X\right)_{\pi} \rightarrow T X$ to be $\rho=\Phi^{-1} \circ \pi^{\#} \circ \Psi$ and the bracket

$$
[\alpha, \beta]_{\pi}=L_{\rho \alpha} \beta-L_{\rho \beta} \alpha-\mathrm{d}(\rho \alpha, \beta)
$$

$\forall \alpha, \beta \in \Gamma\left(\left.T^{*} X\right|_{U}\right)$ holomorphic.

Its associated complex Lie algebroid $A^{1,0}$ will be denoted $\left(T^{1,0} X\right)_{\pi}^{*}$ since its underlying complex vector bundle is $\left(T^{1,0} X\right)^{*}$. The following lemma is obvious.

Lemma 3.6. For the associated complex Lie algebroid $\left(T^{1,0} X\right)_{\pi}^{*}$, the anchor map is

$$
\left(T^{1,0} X\right)^{*} \stackrel{\pi^{\sharp}}{\rightarrow} T_{\mathbb{C}} X
$$

and the bracket on $\Omega^{1,0}(X)$ is given by

$$
\left[\xi^{1,0}, \eta^{1,0}\right]=L_{\pi^{\sharp} \xi^{1,0}} \eta^{1,0}-L_{\pi^{\sharp} \eta^{1,0}} \xi^{1,0}-\partial \pi\left(\xi^{1,0}, \eta^{1,0}\right), \quad \forall \xi^{1,0}, \eta^{1,0} \in \Omega^{1,0}(X) .
$$


The following proposition describes the underlying real and imaginary Lie algebroids of $\left(T^{*} X\right)_{\pi}$.

Proposition 3.7. Let $(X, \pi)$ be a holomorphic Poisson manifold, where $\pi=\pi_{R}+i \pi_{I} \in$ $\Gamma\left(\wedge^{2} T^{1,0} X\right)$. Then, the underlying real and imaginary Lie algebroids of $\left(T^{*} X\right)_{\pi}$ are isomorphic to $\left(T^{*} X\right)_{4 \pi_{R}}$ and $\left(T^{*} X\right)_{4 \pi_{I}}$, respectively.

Proof. First, let us consider the anchor map $\rho:\left(T^{*} X\right)_{\pi} \rightarrow T X$ as a bundle map of real vector bundles. Clearly, we have

$$
\rho=\Phi^{-1} \circ \pi^{\#} \circ \Psi=\Phi^{-1} \circ \pi_{R}^{\#} \circ\left(1-i J^{*}\right) \circ\left(1-i J^{*}\right)=4 \pi_{R}^{\#} .
$$

Now, we consider the bracket. For this purpose, let $A_{R}$ be its underlying real Lie algebroid and $A_{\mathbb{C}}$ the complexification of $A_{R}$. Note that for any holomorphic functions $f, g \in \mathcal{O}_{X}(U)$, we have

$$
[d f, d g]_{\pi}=d\{f, g\},
$$

where both sides are considered as sections of $A_{\mathbb{C}}$. It thus follows, from Corollary 2.4, that

$$
\Re[d f, d g]_{\pi}=d \Re\{f, g\}=4 d\{\Re f, \Re g\}_{R}=4[\mathrm{~d} \Re(f), \mathrm{d} \Re(g)]_{\pi_{R}} .
$$

Therefore, $A_{R}$ is isomorphic to $\left(T^{*} X\right)_{4 \pi_{R}}$.

Finally, note that the Nijenhuis structure $j: A_{R} \rightarrow A_{R}$ in Section 3.3 is simply $J^{*}$. It thus follows that $\left(A_{R}\right)_{j}$ is isomorphic to $\left(T^{*} X\right)_{4 \pi_{I}}$ using Theorem 2.7.

\subsection{Holomorphic Lie-Poisson structures}

Similar to the smooth case, there is also another equivalent definition of holomorphic Lie algebroids. The proof is similar to the smooth case, and is left to the reader. Note that the complex dual $\operatorname{Hom}_{\mathbb{C}}(A, \mathbb{C})$ of a holomorphic vector bundle $A \rightarrow X$ is again a complex manifold, which is also a holomorphic vector bundle over $X$. We denote by $p: \operatorname{Hom}_{\mathbb{C}}(A, \mathbb{C}) \rightarrow X$ the projection onto the base manifold. There is a one-one correspondence between holomorphic sections $V \in \mathcal{A}(U)$ and fiberwise-linear holomorphic 
functions $l_{V}$ on $\operatorname{Hom}_{\mathbb{C}}\left(\left.A\right|_{U}, \mathbb{C}\right): \forall \alpha \in \operatorname{Hom}_{\mathbb{C}}\left(\left.A\right|_{U}, \mathbb{C}\right)$

$$
l_{V}(\alpha)=\alpha\left(\left.V\right|_{p(\alpha)}\right)
$$

Proposition 3.8. Let $A \rightarrow X$ be a holomorphic vector bundle. The following are equivalent:

(a) $A$ is a holomorphic Lie algebroid;

(b) there exists a fiberwise-linear holomorphic Poisson structure on $\operatorname{Hom}_{\mathbb{C}}(A, \mathbb{C})$. Here the Lie algebroid structure on $(A, \rho,[\cdot, \cdot])$ and the Poisson structure on $\operatorname{Hom}_{\mathbb{C}}(A, \mathbb{C})$ are related by the following equations:

$$
\begin{aligned}
\left\{p^{*} f, l_{V}\right\} & =p^{*}(\rho(V)(f)) \\
\left\{l_{V}, l_{W}\right\} & =l_{[V, W]}
\end{aligned}
$$

for any $V, W \in \mathcal{A}(U)$ and $f \in \mathcal{O}_{X}(U)$, where $p: \operatorname{Hom}_{\mathbb{C}}(A, \mathbb{C}) \rightarrow X$ is the projection.

Summarizing the discussions above, we get the main result of this section.

Theorem 3.9. Let $A \rightarrow X$ be a holomorphic vector bundle. There is a one-to-one correspondence between:

(a) holomorphic Lie algebroid structures on $A \rightarrow X$,

(b) fiberwise-linear holomorphic Poisson structures on $\operatorname{Hom}_{\mathbb{C}}(A, \mathbb{C})$,

\subsection{Real and imaginary parts of a holomorphic Lie-Poisson structure}

Any complex vector space $V$ can be equivalently thought of as a real vector space with an $\mathbb{R}$-linear endomorphism $j$, such that $j^{2}=-1$, representing the multiplication by the imaginary number $\sqrt{-1}$.

Given a complex vector space $V$, its complex dual space is the set of morphisms $\operatorname{Hom}_{\mathbb{C}}(V, \mathbb{C})$ from $V$ to $\mathbb{C}$ in the category of complex vector spaces. Similarly, its real dual space is the set of morphisms $\operatorname{Hom}_{\mathbb{R}}(V, \mathbb{R})$ from $V$ to $\mathbb{R}$ in the category of real vector spaces. Clearly, $\operatorname{Hom}_{\mathbb{C}}(V, \mathbb{C})$ is a vector space over $\mathbb{C}$ while $\operatorname{Hom}_{\mathbb{R}}(V, \mathbb{R})$ is a vector space over $\mathbb{R}$. 
The map

$$
\operatorname{Hom}_{\mathbb{R}}(V, \mathbb{R}) \rightarrow \operatorname{Hom}_{\mathbb{C}}(V, \mathbb{C}): f \mapsto\left(1-i j^{*}\right) f
$$

is an isomorphism of real vector spaces. Indeed, $g \in \operatorname{Hom}_{\mathbb{C}}(V, \mathbb{C})$ if and only if $g=(1-$ $\left.i j^{*}\right) f$ with $f(=\Re \circ g) \in \operatorname{Hom}_{\mathbb{R}}(V, \mathbb{R})$.

Given a complex vector bundle $A \rightarrow X$, we denote its complex and real dual bundles by $\operatorname{Hom}_{\mathbb{C}}(A, \mathbb{C}) \rightarrow X$ and $\operatorname{Hom}_{\mathbb{R}}(A, \mathbb{R}) \rightarrow X$, respectively. Applying the previous isomorphism fiberwise yields the isomorphism of real vector bundles $\Psi=1-i j^{*}$ :

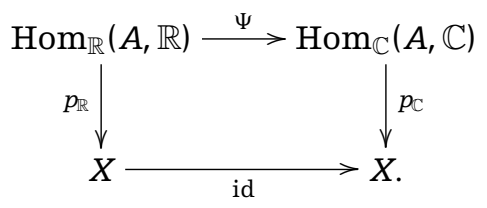

Here $p_{\mathbb{C}}$ and $p_{\mathbb{R}}$ denote the projections of the vector bundles $\operatorname{Hom}_{\mathbb{C}}(A, \mathbb{C}), \operatorname{Hom}_{\mathbb{R}}(A, \mathbb{R})$ onto their base $X$. Note that $\Psi^{-1}(\xi)=\Re \circ \xi$.

We consider a holomorphic Lie algebroid $(A \rightarrow X, \rho,[\cdot, \cdot])$. According to Proposition 3.8, the complex dual bundle $\operatorname{Hom}_{\mathbb{C}}(A, \mathbb{C})$ is a fiberwise linear holomorphic Poisson manifold, whose holomorphic Poisson tensor is denoted by $\pi$. Let $\pi_{R}$ and $\pi_{I}$ be its real and imaginary parts. Then, $\pi_{\Re}:=\Psi_{*}^{-1} \pi_{R}$ and $\pi_{\Im}:=\Psi_{*}^{-1} \pi_{I}$ are fiberwise $\mathbb{R}$-linear Poisson tensors on the real dual bundle $\operatorname{Hom}_{\mathbb{R}}(A, \mathbb{R})$. These Poisson structures therefore correspond to real Lie algebroids on $A \rightarrow X$, which are denoted by $\left(A \rightarrow X, \rho_{\Re},[\cdot, \cdot]_{\Re}\right)$ or $A_{\Re}$ and $\left(A \rightarrow X, \rho_{\Im},[\cdot, \cdot]_{\Im}\right)$ or $A_{\Im}$, respectively. The following Proposition identifies these Lie algebroids with those discussed in Section 3.3.

Proposition 3.10. Let $(A \rightarrow X, \rho,[\cdot, \cdot])$ be a holomorphic Lie algebroid.

(a) The Lie algebroid $\left(A \rightarrow X, 4 \rho_{\Re}, 4[\cdot, \cdot]_{\mathfrak{R}}\right)$ is isomorphic to the real Lie algebroid $A_{R}$;

(b) The Lie algebroid $\left(A \rightarrow X,-4 \rho_{\mathfrak{\Im}},-4[\cdot, \cdot]_{\Im}\right)$ is isomorphic to the imaginary Lie algebroid $A_{I}$.

Proof. First, we fix some notations. Any section $V \in \Gamma(A)$ can be thought of as a fiberwise $\mathbb{C}$-linear (respectively $\mathbb{R}$-linear) function on $\operatorname{Hom}_{\mathbb{C}}(A, \mathbb{C})\left(\operatorname{respectively} \operatorname{Hom}_{\mathbb{R}}(A, \mathbb{R})\right.$ ), which we denote by $l_{V}$ (resp. $l_{V}^{\prime}$ ). 
For all $V \in \Gamma(A)$ and $f \in \operatorname{Hom}_{\mathbb{R}}(A, \mathbb{R})$, we have

$$
\left(\Psi^{*} l_{V}\right)(f)=l_{V}\left(f-i\left(j^{*} f\right)\right)=f(V)-i f(j V)=l_{V}^{\prime}(f)-i l_{j V}^{\prime}(f) .
$$

Thus

$$
\left\{\begin{array}{l}
\Re\left(\Psi^{*} l_{V}\right)=l_{V}^{\prime} \\
\Im\left(\Psi^{*} l_{V}\right)=-l_{j V}^{\prime}
\end{array}\right.
$$

First, we look at the anchor map $\rho_{\Re}$. Given any $x \in X, \alpha \in A_{X}$, and $\eta \in T_{X}^{*} X$, to compute $\left\langle\eta, \rho_{\Re}(\alpha)\right\rangle$, we choose an open neighborhood $U \subset X$ containing $X$, a holomorphic section $V \in \mathcal{A}(U)$ through $\alpha$, and a holomorphic function $f \in \mathcal{O}_{X}(U)$ with $\left.\mathrm{d}(\Re(f))\right|_{X}=\eta$.

Consider the relation

$$
p_{\mathbb{C}}^{*}(\rho(V) f)=\left\{p_{\mathbb{C}}^{*} f, l_{V}\right\}
$$

from Proposition 3.8. Taking the real part, we obtain, by Corollary 2.4:

$$
p_{\mathbb{C}}^{*}(\rho(V) \Re(f))=4\left\{p_{\mathbb{C}}^{*} \Re(f), \Re\left(l_{V}\right)\right\}_{R}
$$

Applying $\Psi^{*}$ to both sides and using equations (10) and (11), we have

$$
\begin{aligned}
p_{\mathbb{R}}^{*}(\rho(V) \Re(f))=\Psi^{*} p_{\mathbb{C}}^{*}(\rho(V) \Re(f))=4 \Psi^{*}\left\{p_{\mathbb{C}}^{*} \Re(f), \Re\left(l_{V}\right)\right\}_{R} \\
=4\left\{\Psi^{*} p_{\mathbb{C}}^{*} \Re(f), \Re\left(\Psi^{*} l_{V}\right)\right\}_{\Re}=4\left\{p_{\mathbb{R}}^{*} \Re(f), l_{V}^{\prime}\right\}_{\Re}=4 p_{\mathbb{R}}^{*}\left(\rho_{\Re}(V) \Re(f)\right) .
\end{aligned}
$$

Hence, it follows that

$$
\rho(V) \Re(f)=4 \rho_{\Re}(V) \Re(f)
$$

and

$$
\left\langle\eta, 4 \rho_{\Re}(\alpha)\right\rangle=\langle\eta, \rho(\alpha)\rangle
$$

We now identify the anchor map $\rho_{\Im}$. Taking the imaginary part of the relation

$$
p_{\mathbb{C}}^{*}(\rho(V) f)=\left\{p_{\mathbb{C}}^{*} f, l_{V}\right\}
$$


from Proposition 3.8, we obtain

$$
p_{\mathbb{C}}^{*}(\rho(V) \Im(f))=\Im\left\{p_{\mathbb{C}}^{*} f, l_{V}\right\}=-4\left\{p_{\mathbb{C}}^{*} \mathfrak{I}(f), \Im\left(l_{V}\right)\right\}_{I}
$$

by Corollary 2.4. Applying $\Psi^{*}$ to both sides and making use of equations (10) and (11), we get:

$$
\begin{aligned}
p_{\mathbb{R}}^{*}(\rho(V) \Im(f))=\Psi^{*} p_{\mathbb{C}}^{*}(\rho(V) \Im(f)) & =-4 \Psi^{*}\left\{p_{\mathbb{C}}^{*} \Im(f), \Im\left(l_{V}\right)\right\}_{I} \\
& =-4\left\{\Psi^{*} p_{\mathbb{C}}^{*} \Im(f), \Psi^{*}\left(l_{V}\right)\right\}_{\Im}=4\left\{p_{\mathbb{R}}^{*} \Im(f), l_{j V}^{\prime}\right\}_{\Im}
\end{aligned}
$$

It follows that

$$
\rho(V) \Im(f)=4 \rho_{\Im}(j V) \Im(f)
$$

which implies that $4 \rho_{\Im}=-\rho_{j}$.

We now consider the Lie brackets. Choose an open neighborhood $U \subset X$ such that the holomorphic vector bundle $\left.A\right|_{U} \rightarrow U$ is trivial. Since the module of smooth sections of $\left.A\right|_{U}$ is generated (over $C^{\infty}(U, \mathbb{R})$ ) by the holomorphic sections, it suffices to show that the bracket $4[V, W]_{\Re}$ (respectively $-4[V, W]_{\mathfrak{S}}$ ) is equal to $[V, W]$ (respectively $[V, W]_{j}$ ), for any two holomorphic sections $V, W \in \mathcal{A}(U)$.

According to Proposition 3.8, we have

$$
\left\{l_{V}, l_{W}\right\}=l_{[V, W]}, \quad \forall V, W \in \mathcal{A}(U) .
$$

By Corollary 2.4, we obtain

$$
\Re\left(l_{[V, W]}\right)=\Re\left\{l_{V}, l_{W}\right\}=4\left\{\Re\left(l_{V}\right), \Re\left(l_{W}\right)\right\}_{R}
$$

Therefore, applying $\Psi^{*}$ to both sides, we get

$$
\Re\left(\Psi^{*} l_{[V, W]}\right)=4 \Psi^{*}\left\{\Re\left(l_{V}\right), \Re\left(l_{W}\right)\right\}_{R}=4\left\{\Re\left(\Psi^{*} l_{V}\right), \Re\left(\Psi^{*} l_{W}\right)\right\}_{\Re}
$$

and, using (11), we obtain

$$
l_{[V, W]}^{\prime}=4\left\{l_{V}^{\prime}, l_{W}^{\prime}\right\}_{\Re}
$$

Hence, it follows that $4[V, W]_{\Re}=[V, W]$. 
We now turn our attention to the Lie bracket $[\cdot, \cdot]_{\mathfrak{S}}$. Taking the imaginary part of equation (12) and applying $\Psi^{*}$ to both sides, it follows from Corollary 2.4 that

$$
-4\left\{\Im\left(\Psi^{*} l_{V}\right), \Im\left(\Psi^{*} l_{W}\right)\right\}_{\Im}=\Im\left(\Psi^{*} l_{[V, W]}\right) .
$$

Hence, using equation (11), we obtain

$$
4 l_{[j V, j W]_{\Im}}^{\prime}=4\left\{l_{j V}^{\prime}, l_{j W}^{\prime}\right\}_{\Im}=l_{j[V, W]}^{\prime}
$$

Therefore,

$$
4[j V, j W]_{\Im}=j[V, W]
$$

and

$$
[V, W]_{j}=j^{-1}[j V, j W]=-4[V, W]_{\Im}
$$

This completes the proof.

Remark 3.11. In particular, given a Lie algebra $\mathfrak{g}$ over $\mathbb{C}$, its complex dual $\operatorname{Hom}_{\mathbb{C}}(\mathfrak{g}, \mathbb{C})$ is a holomorphic Poisson manifold. The isomorphism $\operatorname{Hom}_{\mathbb{R}}(\mathfrak{g}, \mathbb{R}) \stackrel{\Psi}{\rightarrow} \operatorname{Hom}_{\mathbb{C}}(\mathfrak{g}, \mathbb{C})$ maps the Lie-Poisson structure on $\operatorname{Hom}_{\mathbb{R}}(\mathfrak{g}, \mathbb{R})$ corresponding to the Lie algebra bracket $v \otimes w \mapsto$ $\frac{1}{4}[v, w]$ (respectively $v \otimes w \mapsto-\frac{1}{4} j[v, w]$ ) on $\mathfrak{g}$ to the real (respectively imaginary) part of the holomorphic Poisson structure on $\operatorname{Hom}_{\mathbb{C}}(\mathfrak{g}, \mathbb{C})$. Here, $j: \mathfrak{g} \rightarrow \mathfrak{g}$ is the $\mathbb{R}$-linear operator representing the scalar multiplication by $\sqrt{-1} \in \mathbb{C}$.

This is an immediate consequence of the relations

$$
\left\{l_{V}^{\prime}, l_{W}^{\prime}\right\}_{\Re}=l_{\frac{1}{4}[V, W]}^{\prime} \quad\left\{l_{V}^{\prime}, l_{W}^{\prime}\right\}_{\Im}=l_{-\frac{1}{4}[V, W]_{j}}^{\prime}
$$

and the following fact: since here, the holomorphic vector bundle $A \rightarrow X$ is reduced to the vector space $\mathfrak{g}$, all elements $V, W$ of $\mathfrak{g}$ are automatically holomorphic sections and we have

$$
[V, W]_{j}=[j V, W]+[V, j W]-j[V, W]=j[V, W]
$$

since the restriction of the Lie bracket to the holomorphic sections is $\mathbb{C}$-linear. 


\section{Holomorphic Lie Algebroid Cohomology and Holomorphic Poisson Cohomology}

\subsection{Matched pairs}

The notion of matched pairs of Lie algebroids was introduced by Lu in her classification of Poisson group actions [31], and further studied by Mokri [36] and Mackenzie [32].

Definition 4.1. A matched pair of Lie algebroids is a pair of (complex or real) Lie algebroids $A$ and $B$ over the same base manifold $M$, where $B$ is an $A$-module and $A$ is a $B$-module such that the following identities hold:

$$
\begin{gathered}
{[a(X), b(Y)]=-a\left(\nabla_{Y} X\right)+b\left(\nabla_{X} Y\right),} \\
\nabla_{X}\left[Y_{1}, Y_{2}\right]=\left[\nabla_{X} Y_{1}, Y_{2}\right]+\left[Y_{1}, \nabla_{X} Y_{2}\right]+\nabla_{\nabla_{Y_{2}} X} Y_{1}-\nabla_{\nabla_{Y_{1}} X} Y_{2}, \\
\nabla_{Y}\left[X_{1}, X_{2}\right]=\left[\nabla_{Y} X_{1}, X_{2}\right]+\left[X_{1}, \nabla_{Y} X_{2}\right]+\nabla_{\nabla_{X_{2}} Y} X_{1}-\nabla_{\nabla_{X_{1}} Y} X_{2},
\end{gathered}
$$

where $X_{1}, X_{2}, X \in \Gamma(A)$ and $Y_{1}, Y_{2}, Y \in \Gamma(B)$. Here, $a$ and $b$ are the anchor maps of $A$ and $B$, respectively, and $\nabla$ denotes both the representation

$$
\Gamma(A) \otimes \Gamma(B) \rightarrow \Gamma(B):(X, Y) \mapsto \nabla_{X} Y
$$

of $A$ on $B$ and the representation

$$
\Gamma(B) \otimes \Gamma(A) \rightarrow \Gamma(A):(Y, X) \mapsto \nabla_{Y} X
$$

of $B$ on $A$.

Theorem 4.2 ([32, 36]). Given a matched pair $(A, B)$ of Lie algebroids, there is a Lie algebroid structure $A \bowtie B$ on the direct sum vector bundle $A \oplus B$, with anchor $c(X \oplus Y)=$ $a(X)+b(Y)$ and bracket

$$
\left[X_{1} \oplus Y_{1}, X_{2} \oplus Y_{2}\right]=\left(\left[X_{1}, X_{2}\right]+\nabla_{Y_{1}} X_{2}-\nabla_{Y_{2}} X_{1}\right) \oplus\left(\left[Y_{1}, Y_{2}\right]+\nabla_{X_{1}} Y_{2}-\nabla_{X_{2}} Y_{1}\right)
$$

Conversely, if $A \oplus B$ has a Lie algebroid structure for which $A \oplus 0$ and $0 \oplus B$ are Lie subalgebroids, then the representations $\nabla$ defined by

$$
[X \oplus 0,0 \oplus Y]=-\nabla_{Y} X \oplus \nabla_{X} Y
$$

endow the pair $(A, B)$ with a matched pair structure. 
See [32] for more details.

Example 4.3. Let $X$ be a complex manifold. Then, $\left(T^{0,1} X, T^{1,0} X\right)$ is a matched pair, where the actions are given by

$$
\nabla_{X^{0,1}} X^{1,0}=\operatorname{pr}^{1,0}\left[X^{0,1}, X^{1,0}\right] \quad \text { and } \quad \nabla_{X^{1,0}} X^{0,1}=\operatorname{pr}^{0,1}\left[X^{1,0}, X^{0,1}\right]
$$

for all $X^{0,1} \in \mathfrak{X}^{0,1}(X)$ and $X^{1,0} \in \mathfrak{X}^{1,0}(X)$. Hence, $T^{0,1} X \bowtie T^{1,0} X$ and $T_{\mathbb{C}} X$ are isomorphic as complex Lie algebroids.

More generally, given a holomorphic Lie algebroid $A$, the pair $\left(A^{0,1}, A^{1,0}\right)$ is a matched pair of Lie algebroids and $A^{0,1} \bowtie A^{1,0}$ is isomorphic, as a complex Lie algebroid, to $A_{\mathbb{C}}$.

Let $A$ and $B$ be two (complex or real) Lie algebroids over the same base manifold $M$. Assume $B$ is an $A$-module and $A$ is a $B$-module, both representations being abusively denoted by the same symbol $\nabla$. And define

$$
\begin{gathered}
F(X ; Y):=[a(X), b(Y)]+a\left(\nabla_{Y} X\right)-b\left(\nabla_{X} Y\right), \\
S\left(X ; Y_{1}, Y_{2}\right):=\left[\nabla_{X} Y_{1}, Y_{2}\right]+\left[Y_{1}, \nabla_{X} Y_{2}\right]-\nabla_{X}\left[Y_{1}, Y_{2}\right]+\nabla_{\nabla_{Y_{2}} X} Y_{1}-\nabla_{\nabla_{Y_{1}} X} Y_{2}, \\
T\left(Y ; X_{1}, Y_{2}\right):=\left[\nabla_{Y} X_{1}, X_{2}\right]+\left[X_{1}, \nabla_{Y} X_{2}\right]-\nabla_{Y}\left[X_{1}, X_{2}\right]+\nabla_{\nabla_{X_{2}} Y} X_{1}-\nabla_{\nabla_{X_{1}} Y} X_{2},
\end{gathered}
$$

where $a$ and $b$ are the respective anchor maps of $A$ and $B$, while $X_{1}, X_{2}, X \in \Gamma(A)$ and $Y_{1}, Y_{2}, Y \in \Gamma(B)$.

The following result can be verified directly.

Lemma 4.4. For any (complex or real-valued) function $f$ on $M$, we have

$$
\begin{aligned}
F(f X ; Y) & =f F(X ; Y) & F(X ; f Y) & =f F(X ; Y), \\
S\left(f X ; Y_{1}, Y_{2}\right) & =f S\left(X ; Y_{1}, Y_{2}\right) & T\left(f Y ; X_{1}, X_{2}\right) & =f T\left(Y ; X_{1}, X_{2}\right),
\end{aligned}
$$

and

$$
\begin{aligned}
S\left(X ; f Y_{1}, Y_{2}\right) & =f S\left(X ; Y_{1}, Y_{2}\right)+F\left(X ; Y_{2}\right)(f) Y_{1} \\
T\left(Y ; f X_{1}, X_{2}\right) & =f T\left(Y ; X_{1}, X_{2}\right)-F\left(X_{2} ; Y\right)(f) X_{1}
\end{aligned}
$$

Moreover, $S$ and $T$ are skew symmetric in their last two arguments. 


\subsection{Cohomology of a matched pair}

Proposition 4.5. Let $A$ and $B$ be a pair of Lie algebroids over $M$ with mutual actions $\nabla$. The pair $(A, B)$ is a matched pair iff the diagram

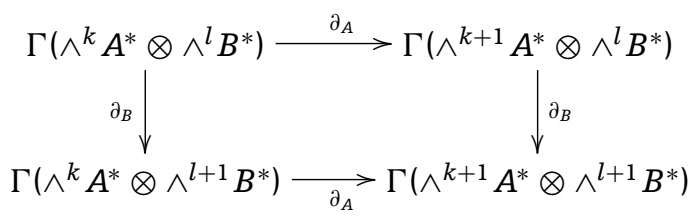

commutes, where $\partial_{A}$ and $\partial_{B}$ denote the Lie algebroid cohomology differential operators of $A$ with values in the module $\wedge^{\bullet} B^{*}$ and of $B$ with values in the module $\wedge^{\bullet} A^{*}$, respectively.

Here, if $\alpha \in \Gamma\left(\wedge^{k} A^{*} \otimes \wedge^{l} B^{*}\right)$, then $\partial_{A}$ and $\partial_{B}$ are given by

$$
\begin{aligned}
& \left(\partial_{A} \alpha\right)\left(A_{0}, \ldots, A_{k}, B_{1}, \ldots, B_{l}\right) \\
= & \sum_{i=0}^{k}(-1)^{i}\left(a\left(A_{i}\right) \alpha\left(A_{0}, \ldots, \widehat{A}_{i}, \ldots, A_{k}, B_{1}, \ldots, B_{l}\right)\right. \\
& \left.-\sum_{j=1}^{l} \alpha\left(A_{0}, \ldots, \widehat{A}_{i}, \ldots, A_{k}, B_{1}, \ldots, \nabla_{A_{i}} B_{j}, \ldots, B_{l}\right)\right) \\
& +\sum_{i<j}(-1)^{i+j} \alpha\left(\left[A_{i}, A_{j}\right], A_{0}, \ldots, \widehat{A}_{i}, \ldots, \widehat{A_{j}}, \ldots, A_{k}, B_{1}, \ldots, B_{l}\right)
\end{aligned}
$$

and

$$
\begin{aligned}
& \left(\partial_{B} \alpha\right)\left(A_{1}, \ldots, A_{k}, B_{0}, \ldots, B_{l}\right) \\
= & \sum_{i=0}^{l}(-1)^{i}\left(b\left(B_{i}\right) \alpha\left(A_{1}, \ldots, A_{k}, B_{0}, \ldots, \widehat{B}_{i}, \ldots, B_{l}\right)\right. \\
& \left.-\sum_{j=1}^{k} \alpha\left(A_{1}, \ldots, \nabla_{B_{i}} A_{j}, \ldots, A_{k}, B_{0}, \ldots, \widehat{B}_{i}, \ldots, B_{l}\right)\right) \\
& +\sum_{i<j}(-1)^{i+j} \alpha\left(A_{1}, \ldots, A_{k},\left[B_{i}, B_{j}\right], B_{0}, \ldots, \widehat{B}_{i}, \ldots, \widehat{B_{j}}, \ldots, B_{l}\right) .
\end{aligned}
$$

Proof. $\Rightarrow$ If the pair $(A, B)$ is a matched pair, then the direct sum $A \oplus B$ is a Lie algebroid with bracket given by equation (16). And the corresponding Lie algebroid differential

$$
\Gamma\left(\wedge^{\bullet}(A \oplus B)^{*}\right) \stackrel{d_{A \bowtie B}}{\longrightarrow} \Gamma\left(\wedge^{\bullet+1}(A \oplus B)^{*}\right),
$$


defined by

$$
\begin{aligned}
\left(d_{A \bowtie B} \alpha\right)\left(C_{0}, \ldots, C_{n}\right)= & \sum_{i=0}^{n}(-1)^{i} c\left(C_{i}\right)\left(\alpha\left(C_{0}, \ldots, \widehat{C}_{i}, \ldots, C_{n}\right)\right) \\
& +\sum_{i<j}(-1)^{i+j} \alpha\left(\left[C_{i}, C_{j}\right], C_{0}, \ldots, \widehat{C}_{i}, \ldots, \widehat{C_{j}}, \ldots, C_{n}\right)
\end{aligned}
$$

satisfies $d_{A \bowtie B}^{2}=0$. Now, remember that

$$
\wedge^{n}(A \oplus B)^{*}=\bigoplus_{k+l=n} \wedge^{k} A^{*} \otimes \wedge^{l} B^{*}
$$

It is easy to see that

$$
\begin{aligned}
d_{A \bowtie B}\left(\Gamma\left(\wedge^{k} A^{*} \otimes \wedge^{l} B^{*}\right)\right) \subset \Gamma\left(\wedge^{k+2} A^{*} \otimes \wedge^{l-1} B^{*}\right) & \oplus \Gamma\left(\wedge^{k+1} A^{*} \otimes \wedge^{l} B^{*}\right) \\
& \oplus \Gamma\left(\wedge^{k} A^{*} \otimes \wedge^{l+1} B^{*}\right) \oplus \Gamma\left(\wedge^{k-1} A^{*} \otimes \wedge^{l+2} B^{*}\right) .
\end{aligned}
$$

Moreover, since $A$ and $B$ are Lie subalgebroids of $A \bowtie B$, the stronger relation

$$
d_{A \bowtie B} \Gamma\left(\wedge^{k} A^{*} \otimes \wedge^{l} B^{*}\right) \subset \Gamma\left(\wedge^{k+1} A^{*} \otimes \wedge^{l} B^{*}\right) \oplus \Gamma\left(\wedge^{k} A^{*} \otimes \wedge^{l+1} B^{*}\right)
$$

holds. Composing $d_{A \bowtie B}$ with the natural projections on each of the direct summands, we get the commutative diagram as

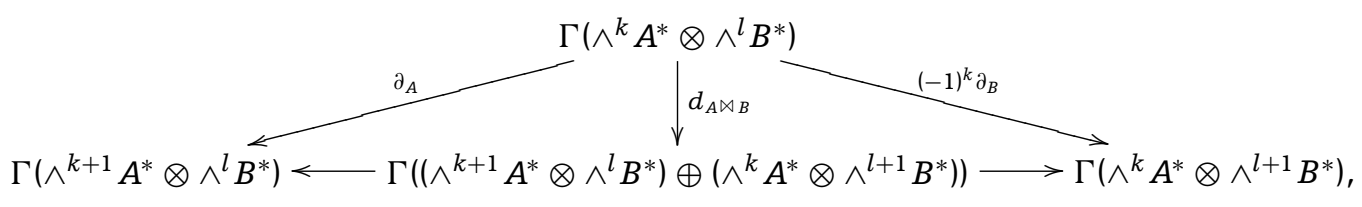

where $\partial_{A}$ and $\partial_{B}$ are the coboundary operators given by equations (18) and (19). From $d_{A \bowtie B}^{2}=0$, it follows that $\partial_{A}^{2}=0, \partial_{B}^{2}=0$, and $\partial_{A} \circ \partial_{B}=\partial_{B} \circ \partial_{A}$. $\Leftarrow$ Conversely, given the commutative diagram (17), one can define an operator

$$
\Gamma\left(\wedge^{\bullet}(A \oplus B)^{*}\right) \stackrel{d_{A \bowtie B}}{\longrightarrow} \Gamma\left(\wedge^{\bullet+1}(A \oplus B)^{*}\right),
$$

whose restriction to $\Gamma\left(\wedge^{k} A^{*} \otimes \wedge^{l} B^{*}\right)$ is $\partial_{A}+(-1)^{k} \partial_{B}$. Clearly, $d_{A \bowtie B}^{2}=0$ and $\left(\Gamma\left(\wedge^{\bullet}(A \oplus\right.\right.$ $\left.\left.B)^{*}\right), d_{A \bowtie B}\right)$ is a differential graded algebra. Hence, it follows that $A \oplus B$ admits a Lie 
algebroid structure with associated differential $d_{A \bowtie B}$. Moreover,

$$
d_{A \bowtie B} \Gamma\left(\wedge^{k} A^{*} \otimes \wedge^{l} B^{*}\right) \subset \Gamma\left(\wedge^{k+1} A^{*} \otimes \wedge^{l} B^{*}\right) \oplus \Gamma\left(\wedge^{k} A^{*} \otimes \wedge^{l+1} B^{*}\right) .
$$

Therefore, $\Gamma(A)$ and $\Gamma(B)$ are closed under the Lie algebroid bracket on $A \oplus B$. The subbundles $A$ and $B$ are thus Lie subalgebroids of $A \oplus B$. We conclude that the pair $(A, B)$ is a matched pair of Lie algebroids.

Proposition 4.6. The Lie algebroid cohomology of $A \bowtie B$ (with trivial coefficients) is isomorphic to the total cohomology of the double complex (17).

Proof. This is an immediate consequence of the following fact, which was already pointed out in the proof of Proposition 4.5: the restriction of the cohomology operator

$$
\Gamma\left(\wedge^{\bullet}(A \oplus B)^{*}\right) \stackrel{d_{A \bowtie B}}{\longrightarrow} \Gamma\left(\wedge^{\bullet+1}(A \oplus B)^{*}\right)
$$

to the subspace $\Gamma\left(\wedge^{k} A^{*} \otimes \wedge^{l} B^{*}\right)$ of $\Gamma\left(\wedge^{k+l}(A \oplus B)^{*}\right)$ is $\partial_{A}+(-1)^{k} \partial_{B}$.

\subsection{Canonical complex Lie algebroid associated to a holomorphic Lie algebroid}

The following standard result is due to Grothendieck, for instance, see [1, 22].

Lemma 4.7. Let $E$ be a complex vector bundle over a complex manifold $X$. Then, $E$ is a holomorphic vector bundle if and only if $E$ is a $T^{0,1} X$-module-i.e. there exists a flat $T^{0,1} X$-connection on $E$ :

$$
\Gamma\left(T^{0,1} X\right) \otimes \Gamma(E) \rightarrow \Gamma(E):(X, \varepsilon) \mapsto \nabla_{X} \varepsilon
$$

Proof. $\Rightarrow$ Let $\mathcal{E}$ denote the sheaf of holomorphic sections of $E \rightarrow X$. For all $U \subset X$ open and $\sigma \in \mathcal{E}(\mathrm{U})$, set $\nabla_{X} \sigma=0, \forall X \in \Gamma\left(\left.T^{0,1} X\right|_{U}\right)$. Then, $\nabla$ extends to all smooth sections of $E$ by

$$
\nabla_{X}(f \varepsilon)=X(f) \varepsilon+f \nabla_{X} \varepsilon
$$

since $\Gamma\left(\left.E\right|_{U}\right)$ is generated by $\mathcal{E}(U)$ over $C^{\infty}(U, \mathbb{C})$. One easily sees that $\nabla$ is a flat $T^{0,1} X$ connection. 
$\Leftarrow$ Let $\nabla$ denote the representation of $T^{0,1} X$ on $E$. And define the sheaf $\mathcal{E}$ over $X$ by

$$
\mathcal{E}(U)=\left\{\sigma \in \Gamma\left(\left.E\right|_{U}\right) \mid \nabla_{X} \sigma=0, \forall X \in \Gamma\left(\left.T^{0,1} X\right|_{U}\right)\right\}
$$

for all $U \subset X$ open. If $\sigma \in \mathcal{E}(U)$, then

$$
\nabla_{X}(f \sigma)=X(f) \sigma+f \nabla_{X} \sigma=X(f) \sigma
$$

for all $X \in \Gamma\left(\left.T^{0,1} X\right|_{U}\right)$. Hence $f \sigma \in \mathcal{E}(U)$ if $f \in \mathcal{O}_{X}(U)$. Thus, $\mathcal{E}$ is a sheaf of $\mathcal{O}_{X}$-modules. Since $\nabla$ is flat, given any $e \in E_{X}$, there exists a neighborhood $U$ of $x$ and a local section $\sigma \in \mathcal{E}(U)$ through $e$. Hence, there exists a holomorphic vector bundle structure on $E$ with $\mathcal{E}$ as sheaf of holomorphic sections.

Now, we can state the main result of this section.

Theorem 4.8. Let $A$ be a holomorphic Lie algebroid over a complex manifold $X$. Then, the pair $\left(T^{0,1} X, A^{1,0}\right)$ is naturally a matched pair of complex Lie algebroids. Conversely, given a complex manifold $X$ and a matched pair $\left(T^{0,1} X, B\right)$, where $B$ is a complex Lie algebroid over $X$ whose anchor takes its values in $T^{1,0} X$, there exists a holomorphic Lie algebroid $A$ such that $B \cong A^{1,0}$ as complex Lie algebroids.

Proof. $\Rightarrow$ Let $\rho$ denote the anchor map of $A$. Since $A$ is a holomorphic vector bundle, by Lemma 4.7, the complex vector bundle $A^{1,0}$ is a $T^{0,1} X$-module. This gives a representation

$$
\Gamma\left(T^{0,1} X\right) \otimes \Gamma\left(A^{1,0}\right) \rightarrow \Gamma\left(A^{1,0}\right):(X, \eta) \mapsto \nabla_{X} \eta
$$

of $T^{0,1} X$ on $A^{1,0}$. On the other hand, the $\mathbb{C}$-linear extension of the anchor map $\rho: A \rightarrow T X$ induces a complex vector bundle map $\rho: A^{1,0} \rightarrow T^{1,0} X$, which is a morphism of complex Lie algebroids. Similar to the situation of Example 4.3, the map

$$
\Gamma\left(A^{1,0}\right) \otimes \Gamma\left(T^{0,1} X\right) \rightarrow \Gamma\left(T^{0,1} X\right):(\eta, X) \mapsto \nabla_{\eta} X:=\operatorname{pr}^{0,1}\left[\rho_{\mathbb{C}}(\eta), X\right]
$$

is automatically a representation of $A^{1,0}$ on $T^{0,1} X$.

It remains to prove that the pair $\left(T^{0,1} X, A^{1,0}\right)$, with the above two representations, satisfies the matched pair axioms (13)-(15). 
If $\eta$ is a holomorphic section of $\left.A^{1,0}\right|_{U}$, then $\nabla_{X} \eta=0$ for all $X \in \Gamma\left(\left.T^{0,1} X\right|_{U}\right.$ ) (by definition of the $T^{0,1} X$-module structure of $\left.A^{1,0}\right)$ and, since $\rho_{\mathbb{C}}(\eta)$ is a holomorphic section of $T^{1,0} X$ over $U$, we have

$$
\operatorname{pr}^{1,0}\left[X, \rho_{\mathbb{C}}(\eta)\right]=0, \quad \forall X \in \Gamma\left(\left.T^{0,1} X\right|_{U}\right)
$$

Thus,

$$
\left[X, \rho_{\mathbb{C}}(\eta)\right]=-\operatorname{pr}^{0,1}\left[\rho_{\mathbb{C}}(\eta), X\right]+\rho_{\mathbb{C}}\left(\nabla_{X} \eta\right),
$$

for all $\eta \in \mathcal{A}^{1,0}(U)$ and $X \in \Gamma\left(T^{0,1} X\right)$. From Lemma 4.4, it follows that equation (21) actually holds for all smooth sections $\eta$ of $A^{1,0}$. This relation is nothing but axiom (13) in the particular case $\left(T^{0,1} X, A^{1,0}\right)$.

If the sections of $A^{1,0}$ involved in equation (14) are taken holomorphic, then equation (14) holds because, in that particular case, all its terms vanish. Therefore, by Lemma 4.4 and the fact that $\mathcal{A}_{\infty}^{1,0}$ is generated by $\mathcal{A}^{1,0}$ as a sheaf of modules over the sheaf $C_{X}^{\infty}$ of smooth functions, equation (14) is always satisfied.

Finally, it follows from the definition of the $A^{1,0}$-module structure on $T^{0,1} X$ and the Jacobi identity that

$$
\nabla_{\eta}\left[X_{1}, X_{2}\right]=\left[\nabla_{\eta} X_{1}, X_{2}\right]+\left[X_{1}, \nabla_{\eta} X_{2}\right], \quad \forall \eta \in \mathcal{A}^{1,0}(U), \forall X_{1}, X_{2} \in \Gamma\left(T^{0,1} X\right)
$$

Hence, equation (15) follows from Lemma 4.4.

$\Leftarrow$ Let $E \rightarrow X$ denote the underlying complex vector bundle, $[\cdot, \cdot]_{B}$ the Lie bracket on $\Gamma(E)$, and $\rho: E \rightarrow T^{1,0} X$ the anchor map of the Lie algebroid $B$. Since $B$ is a $T^{0,1} X$ module, it follows from Lemma 4.7 that $E$ is a holomorphic vector bundle-a smooth section $\eta \in \Gamma\left(\left.E\right|_{U}\right)$ being holomorphic iff $\nabla_{X} \eta=0, \forall X \in \Gamma\left(\left.T^{0,1} X\right|_{U}\right)$.

Moreover, by equation (14), if $\eta_{1}, \eta_{2} \in \Gamma\left(\left.E\right|_{U}\right)$ are two holomorphic sections over an open subset $U \subset X,\left[\eta_{1}, \eta_{2}\right]$ is also a holomorphic section of $E$ over $U$, i.e. the sheaf $\mathcal{E}$ of holomorphic sections of $E$ is a subsheaf of complex Lie subalgebras of the sheaf $\mathcal{E}_{\infty}$ of smooth sections.

We define a new Lie algebroid structure $A$ on the vector bundle $E$ with the composition

$$
E \stackrel{\rho}{\rightarrow} T^{1,0} X \stackrel{2 \Re}{\rightarrow} T X
$$


as anchor map and such that the Lie brackets of $A$ and $B$ agree on the subsheaf $\mathcal{E}$ of $\mathcal{E}_{\infty}$ :

$$
[\sigma, \tau]_{A}=[\sigma, \tau]_{B}, \quad \forall \sigma, \tau \in \mathcal{E}(U)
$$

Here, $\Re$ means real part.

Equation (13) implies that

$$
\nabla_{\eta} Y=\left[\rho_{\mathbb{C}}(\eta), Y\right], \quad \forall \eta \in \mathcal{E}(U), \forall Y \in \Gamma\left(\left.T^{0,1} X\right|_{U}\right)
$$

Thus, $\operatorname{pr}^{1,0}\left[\rho_{\mathbb{C}}(\eta), X\right]=0$. By Example 4.3, $\rho_{\mathbb{C}}(\eta)$ is thus a holomorphic section of $\left.T^{1,0} X\right|_{U}$ if $\eta$ is a holomorphic section of $\left.E\right|_{U}$. Hence, $\rho: E \rightarrow T^{0,1} X$ and $\Re \circ \rho: E \rightarrow T X$ are holomorphic bundle maps.

Note that

$$
f \in \mathcal{O}_{X}(U) \quad \Leftrightarrow \quad d f(X)=2 d f(\Re X), \forall X \in \Gamma\left(T^{1,0} X\right) .
$$

Therefore, the Lie bracket on $\mathcal{A}$ satisfies the Leibniz rule. Indeed, for all $\sigma, \tau \in \mathcal{E}(U)$ and $f \in \mathcal{O}_{X}(U)$, we have

$$
[\sigma, f \tau]_{A}=[\sigma, f \tau]_{B}=d f(\rho(\sigma)) \tau+f[\sigma, \tau]_{B}=d f(2 \Re \circ \rho(\sigma)) \tau+f[\sigma, \tau]_{A} .
$$

Clearly, $A$ is a holomorphic Lie algebroid over $X$ with the same underlying complex vector bundle $E$ and with $\mathcal{E}$ as its sheaf of holomorphic sections. By construction, $A^{1,0}$ and $B$ are isomorphic complex Lie algebroids.

Thus, given a holomorphic Lie algebroid $A \rightarrow X$, we obtain two complex Lie algebroids: $A_{\mathbb{C}}$ and $T^{0,1} X \bowtie A^{1,0}$. The following proposition follows easily from the construction of $T^{0,1} X \bowtie A^{1,0}$.

Proposition 4.9. Assume that $(A \rightarrow X, \rho,[\cdot, \cdot])$ is a holomorphic Lie algebroid. Then,

$$
A_{\mathbb{C}} \rightarrow T^{0,1} X \bowtie A^{1,0}: a \mapsto\left(\rho_{\mathbb{C}}\left(\operatorname{pr}^{0,1}(a)\right), \operatorname{pr}^{1,0}(a)\right)
$$

is a homomorphism of complex Lie algebroids. 


\subsection{Lie algebroid cohomology}

We use the following definition of Lie algebroid cohomology due to Evens-Lu-Weinstein [15].

Definition 4.10. Given a holomorphic Lie algebroid $A \rightarrow X$, let $\Omega_{A}^{k}$ be the sheaf of holomorphic sections of $\wedge^{k} A^{*} \rightarrow X(k=1,2, \ldots)$ and $\Omega_{A}^{0}=\mathcal{O}_{X}$. We have the following complex of sheaves over $X$ :

$$
\Omega_{A}^{\bullet}: \Omega_{A}^{0} \stackrel{d_{A}}{\longrightarrow} \Omega_{A}^{1} \stackrel{d_{A}}{\rightarrow} \cdots \stackrel{d_{A}}{\longrightarrow} \Omega_{A}^{k} \stackrel{d_{A}}{\longrightarrow} \Omega_{A}^{k+1} \stackrel{d_{A}}{\rightarrow} \cdots
$$

where

$$
\begin{aligned}
\left(d_{A} \alpha\right)\left(V_{0}, \ldots, V_{k}\right)= & \sum_{i=0}^{k}(-1)^{i} \rho\left(V_{i}\right) \alpha\left(V_{0}, \ldots, \widehat{V}_{i}, \ldots, V_{k}\right) \\
& +\sum_{i<j}(-1)^{i+j} \alpha\left(\left[V_{i}, V_{j}\right], V_{0}, \ldots, \widehat{V}_{i}, \ldots, \widehat{V}_{j}, \ldots, V_{k}\right)
\end{aligned}
$$

for all $\alpha \in \Omega_{A}^{k}(U), V_{0}, \ldots, V_{k} \in \mathcal{A}(U)$, and any open subset $U$ of $X$.

The holomorphic Lie algebroid cohomology of $A$ (with trivial coefficients) is defined to be the cohomology of the complex of sheaves $\Omega_{A}^{\bullet}$ :

$$
H^{*}(A, \mathbb{C}):=H^{*}\left(X, \Omega_{A}^{\bullet}\right)
$$

The following result gives us an important way of computing holomorphic Lie algebroid cohomology using smooth cohomology, i.e. Lie algebroid cohomology of complex Lie algebroids. In a certain sense, this is a generalization of Dolbeault's theorem to Lie algebroids.

Theorem 4.11. Let $A \rightarrow X$ be a holomorphic Lie algebroid. Then

$$
H^{*}(A, \mathbb{C}) \cong H^{*}\left(T^{0,1} X \bowtie A^{1,0}, \mathbb{C}\right)
$$

where the right-hand side stands for the complex Lie algebroid cohomology of $T^{0,1} X \bowtie A^{1,0}$ (see Proposition 4.6), which can be interpreted as a generalization of the Dolbeault cohomology. 
Proof. By $\Omega_{X}^{0, k} \otimes_{C_{X}^{\infty}} \mathcal{A}_{\infty}^{l, 0}$, we denote the sheaf of sections of the complex vector bundle $\left(T^{0, k} X\right)^{*} \otimes \wedge^{l} A^{1,0} \rightarrow X$. By the holomorphic Poincaré lemma, we have the following resolution of complex of sheaves:

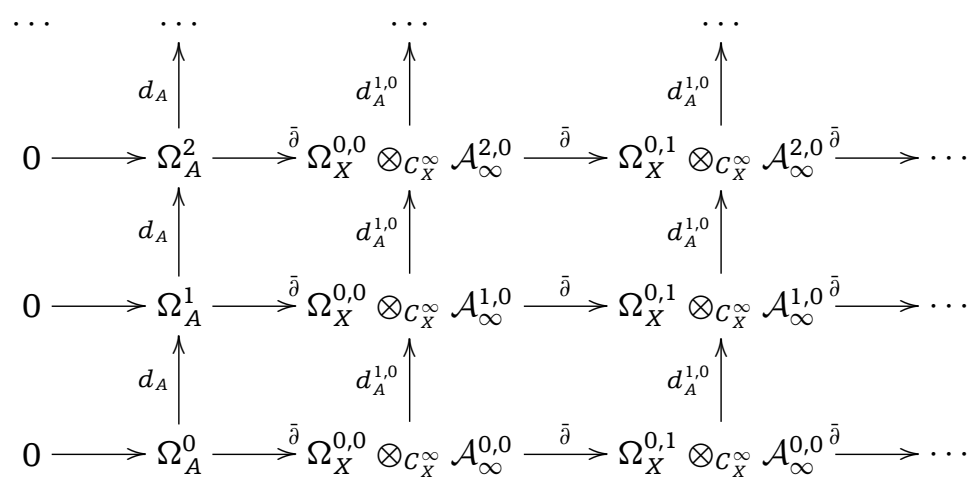

The conclusion thus follows immediately from Theorem 4.8.

Example 4.12. As in Example 4.3, consider a complex manifold $X$. Let $A=T X$ be its holomorphic tangent bundle considered as a holomorphic Lie algebroid. In this particular case, $H^{*}(A, \mathbb{C})$ is the holomorphic de Rham cohomology, while $H^{*}\left(T^{0,1} X \bowtie A^{1,0}, \mathbb{C}\right)$ is the $\mathbb{C}$-valued smooth de Rham cohomology since $T^{0,1} X \bowtie A^{1,0}=T^{0,1} X \bowtie T^{1,0} X \cong T_{\mathbb{C}} X$ as complex Lie algebroids. It is well known that they are isomorphic.

Indeed, the complex Lie algebroid $T^{0,1} X \bowtie A^{1,0}$ is an elliptic Lie algebroid in the sense of Block. Recall that a complex Lie algebroid $(A \rightarrow X,[\cdot, \cdot], \rho)$ is said to be elliptic [1] if the map $\Re \circ \rho: A \rightarrow T X$ is surjective. For an elliptic Lie algebroid, the Lie algebroid cohomology cochain complex is an elliptic complex [1]. Hence, the cohomology groups are finite dimensional if the base manifold is compact. This can also be easily seen directly from our definition of holomorphic Lie algebroid cohomology in terms of a complex of sheaves.

Denote by $H^{*}\left(A_{R}, \mathbb{C}\right)$ the Lie algebroid cohomology of the underlying real Lie algebroid $A_{R}$ with trivial complex coefficients. The following result is an immediate consequence of Theorem 4.11 and Proposition 4.9.

Proposition 4.13. Let $A$ be a holomorphic Lie algebroid with underlying real Lie algebroid $A_{R}$. Then, there is a natural morphism

$$
H^{*}(A, \mathbb{C}) \rightarrow H^{*}\left(A_{R}, \mathbb{C}\right)
$$


Remark 4.14. In [44], Weinstein asked the question how to integrate a complex Lie algebroid. For the complex Lie algebroid $T^{0,1} X \bowtie A^{1,0}$ arising from a matched pair, our discussion above suggests that the holomorphic Lie groupoid integrating the holomorphic Lie algebroid $A$ might be a candidate. It will be interesting to explore further if Theorem 4.8 would have any applications in solving Weinstein's integration problem.

\subsection{Cohomology with general coefficients}

Definition 4.15. Given a holomorphic Lie algebroid $A \rightarrow X$, an $A$-module is a holomorphic vector bundle $E \rightarrow X$ together with a morphism of sheaves (of $\mathbb{C}$-modules)

$$
\mathcal{A} \otimes \mathcal{E} \rightarrow \mathcal{E}: V \otimes s \mapsto \nabla_{V} s
$$

such that, for any open subset $U \subset X$, the relations

$$
\begin{gathered}
\nabla_{f V} s=f \nabla_{V} s \\
\nabla_{V}(f s)=(\rho(V) f) s+f \nabla_{V} s \\
\nabla_{V} \nabla_{W} s-\nabla_{W} \nabla_{V} s=\nabla_{[V, W]} s
\end{gathered}
$$

are satisfied $\forall f \in \mathcal{O}_{X}(U), \forall V, W \in \mathcal{A}(U)$, and $\forall s \in \mathcal{E}(U)$.

Lemma 4.16. Let $A \rightarrow X$ be a holomorphic Lie algebroid and $E \rightarrow X$ a complex vector bundle. Then, $E \rightarrow X$ is an $A$-module if and only if $E \rightarrow X$ is a module over the complex Lie algebroid $T^{0,1} X \bowtie A^{1,0}$.

Proof. $\Rightarrow$ Firstly, note that, since $E \rightarrow X$ is a holomorphic vector bundle with sheaf of holomorphic sections $\mathcal{E}$, by Lemma $4.7, E$ is a $T^{0,1} X$-module whose representation map

$$
\Gamma\left(T^{0,1} X\right) \otimes \Gamma(E) \rightarrow \Gamma(E):(X, s) \mapsto \nabla_{X}^{0,1} s
$$

is entirely characterized by the condition

$$
\mathcal{E}(U)=\left\{\sigma \in \Gamma\left(\left.E\right|_{U}\right) \mid \nabla_{X}^{0,1} \sigma=0, \forall X \in \Gamma\left(\left.T^{0,1} X\right|_{U}\right)\right\}
$$

for all open subsets $U \in X$. 
On the other hand, $E$ is a module over the holomorphic Lie algebroid $(A \rightarrow$ $X,[\cdot, \cdot], \rho)$ with representation map

$$
\mathcal{A} \otimes \mathcal{E} \rightarrow \mathcal{E}:(a, s) \mapsto \nabla_{a} s
$$

where $\mathcal{A}$ and $\mathcal{E}$ are the $\mathcal{O}_{X}$-sheaves of holomorphic sections of $A$ and $E$, respectively. Since $\mathcal{A}_{\infty}$ and $\mathcal{E}_{\infty}$ are generated by $\mathcal{A}$ and $\mathcal{E}$ as sheaves of $C_{X}^{\infty}$-modules, one can define a representation

$$
\Gamma\left(A^{1,0}\right) \otimes \Gamma(E) \rightarrow \Gamma(E):(a, s) \mapsto \nabla_{a}^{1,0} s
$$

of the complex Lie algebroid $\left(A^{1,0},[\cdot, \cdot]^{1,0}, \rho^{1,0}\right)$ on $E$, which is entirely determined by the requirement that, for all open subsets $U$ of $X$, one has

$$
\nabla_{(1-i j) \alpha}^{1,0} \sigma=\nabla_{\alpha} \sigma
$$

for all $\alpha \in \mathcal{A}(U)$ and $\sigma \in \mathcal{E}(U)$.

The equation

$$
\nabla_{(X, a)} s=\nabla_{X}^{0,1} s+\nabla_{a}^{1,0} s
$$

defines a connection of the complex Lie algebroid $T^{0,1} X \bowtie A^{1,0}$ (associated to the holomorphic Lie algebroid $A$ as in Theorem 4.8) on $E$. To check that this covariant derivative is flat, it suffices to prove that

$$
\nabla_{(X, 0)} \nabla_{(0, a)} s-\nabla_{(0, a)} \nabla_{(X, 0)} s=\nabla_{[(X, 0),(0, a)]} s,
$$

for all $X \in \Gamma\left(T^{0,1} X\right), a \in \Gamma\left(A^{1,0}\right)$ and $s \in \Gamma(E)$. However, the curvature being a tensor, it actually suffices to check that, for any open subset $U$ of $X$, one has

$$
\nabla_{(X, 0)} \nabla_{(0,(1-i j) \alpha)} \sigma-\nabla_{(0,(1-i j) \alpha)} \nabla_{(X, 0)} \sigma=\nabla_{[(X, 0),(0,(1-i j) \alpha)]} \sigma
$$

for all $X \in \Gamma\left(\left.T^{0,1} X\right|_{U}\right), \alpha \in \mathcal{A}(U)$, and $\sigma \in \mathcal{E}(U)$. By definition of the Lie algebroid structure of $T^{0,1} X \bowtie A^{1,0}$,

$$
[(X, 0),(0,(1-i j) \alpha)]=\left(-\nabla_{\alpha} X, 0\right)
$$


since $\alpha$ is holomorphic. Hence, equation (23) becomes

$$
\nabla_{X}^{0,1} \nabla_{\alpha} \sigma-\nabla_{\alpha} \nabla_{X}^{0,1} \sigma=\nabla_{-\nabla_{\alpha} X}^{0,1} \sigma
$$

which is obviously true since each term in the equation above vanishes-indeed, the second argument of each $\nabla^{0,1}$ is a holomorphic section of $E$.

$\Leftarrow$ Let $\nabla$ denote the representation of $T^{0,1} X \bowtie A^{1,0}$ on $E$.

Since $T^{0,1} X$ is a Lie subalgebroid of $T^{0,1} X \bowtie A^{1,0}, E$ is a $T^{0,1} X$-module, and thus, by Lemma 4.7, $E \rightarrow X$ is a holomorphic vector bundle whose sheaf of holomorphic sections $\mathcal{E}$ is characterized by

$$
\mathcal{E}(U)=\left\{\sigma \in \Gamma\left(\left.E\right|_{U}\right) \mid \nabla_{(X, 0)} \sigma=0, \forall X \in \Gamma\left(\left.T^{0,1} X\right|_{U}\right)\right\} .
$$

Moreover, the curvature of $\nabla$ being zero, we have

$$
\nabla_{(X, 0)} \nabla_{(0,(1-i j) \alpha)} \sigma-\nabla_{(0,(1-i j) \alpha)} \nabla_{(X, 0)} \sigma=\nabla_{[(X, 0),(0,(1-i j) \alpha)]} \sigma
$$

for any open subset $U$ of $X$ and all $X \in \Gamma\left(\left.T^{0,1} X\right|_{U}\right), \alpha \in \mathcal{A}(U)$, and $\sigma \in \mathcal{E}(U)$. Note that, since $\alpha$ is holomorphic,

$$
[(X, 0),(0,(1-i j) \alpha)] \in \Gamma\left(\left.T^{0,1} X\right|_{U}\right) .
$$

Making use of equations (24) and (26), equation (25) becomes

$$
\nabla_{(X, 0)}\left(\nabla_{(0,(1-i j) \alpha)} \sigma\right)=0
$$

for any open subset $U$ of $X$ and all $X \in \Gamma\left(\left.T^{0,1} X\right|_{U}\right), \alpha \in \mathcal{A}(U)$, and $\sigma \in \mathcal{E}(U)$. In other words, the map

$$
\mathcal{A}(U) \times \mathcal{E}(U) \rightarrow \mathcal{E}(U):(\alpha, \sigma) \mapsto \nabla_{(0,(1-i j) \alpha)} \sigma
$$

does indeed take its values in $\mathcal{E}$. Therefore, this restriction of $\nabla$ endows the holomorphic vector bundle $E \rightarrow X$ with a structure of module over the holomorphic Lie algebroid $A$. 
Definition 4.17. Given a holomorphic Lie algebroid $A \rightarrow X$ and an $A$-module $(E \rightarrow X, \nabla)$, we have the complex of sheaves over $X$

$$
\Omega_{A}^{\bullet} \otimes \mathcal{E}: \Omega_{A}^{0} \otimes \mathcal{E} \stackrel{d_{A}^{\nabla}}{\rightarrow} \Omega_{A}^{1} \otimes \mathcal{E} \stackrel{d_{A}^{\nabla}}{\rightarrow} \cdots \stackrel{d_{A}^{\nabla}}{\rightarrow} \Omega_{A}^{k} \otimes \mathcal{E} \stackrel{d_{A}^{\nabla}}{\rightarrow} \Omega_{A}^{k+1} \otimes \mathcal{E} \stackrel{d_{A}^{\nabla}}{\longrightarrow} \cdots
$$

where

$$
\begin{aligned}
\left(d_{A}^{\nabla} \alpha\right)\left(V_{0}, \ldots, V_{k}\right)= & \sum_{i=0}^{k}(-1)^{i} \nabla_{V_{i}}\left(\alpha\left(V_{0}, \ldots, \widehat{V}_{i}, \ldots, V_{k}\right)\right) \\
& +\sum_{i<j}(-1)^{i+j} \alpha\left(\left[V_{i}, V_{j}\right], V_{0}, \ldots, \widehat{V}_{i}, \ldots, \widehat{V}_{j}, \ldots, V_{k}\right)
\end{aligned}
$$

for any open subset $U$ of $X$ and all $\alpha \in\left(\Omega_{A}^{k} \otimes \mathcal{E}\right)(U)$ and $V_{0}, \ldots, V_{k} \in \mathcal{A}(U)$.

The holomorphic Lie algebroid cohomology of $A$ (with coefficients in the $A$ module $E$ ) is defined to be the cohomology of the complex of sheaves $\Omega_{A}^{\bullet} \otimes \mathcal{E}$ :

$$
H^{*}(A, E):=H^{*}\left(X, \Omega_{A}^{\bullet} \otimes \mathcal{E}\right)
$$

Lemma 4.18. Let $A \rightarrow X$ be a holomorphic Lie algebroid and $E \rightarrow X$ an $A$-module. If $X$ is compact, then $H^{k}(A, E)$ is finite dimensional for all $k$.

The following theorem can be proved in a similar fashion as in Theorem 4.11.

Theorem 4.19. Let $A \rightarrow X$ be a holomorphic Lie algebroid and $E \rightarrow X$ an $A$-module. Then,

$$
H^{*}(A, E) \cong H^{*}\left(T^{0,1} X \bowtie A^{1,0}, E\right)
$$

Given a holomorphic Lie algebroid $A \rightarrow X$ and an $A$-module $E \rightarrow X$, it is simple to see that $E \rightarrow X$ naturally becomes an $A_{R}$-module. The following is a straightforward generalization of Proposition 4.13 .

Proposition 4.20. Let $A$ be a holomorphic Lie algebroid with underlying real Lie algebroid $A_{R}$, and $E \rightarrow X$ an $A$-module. Then, there is a natural homomorphism

$$
H^{*}(A, E) \rightarrow H^{*}\left(A_{R}, E\right)
$$




\subsection{Application to holomorphic Poisson manifolds}

Now, consider the cotangent bundle Lie algebroid $\left(T^{*} X\right)_{\pi}$ associated to a holomorphic Poisson structure $(X, \pi)$. Since $\left(T^{*} X\right)_{\pi}$ is a holomorphic Lie algebroid, according to Theorem 4.8, $\left(T^{0,1} X,\left(T^{1,0} X\right)_{\pi}^{*}\right)$ is a matched pair. On the other hand, according to Theorem 2.7, to any holomorphic Poisson manifold corresponds a natural generalized complex structure. The following theorem indicates the relation between this generalized complex structure and the complex Lie algebroid $T^{0,1} X \bowtie\left(T^{1,0} X\right)_{\pi}^{*}$.

Theorem 4.21. If $(X, \pi)$ is a holomorphic Poisson manifold, then the complex Lie algebroid $T^{0,1} X \bowtie\left(T^{1,0} X\right)_{\pi}^{*}$ is isomorphic to the Dirac structure $L_{4 \pi}$, the $-i$-eigenbundle of the generalized complex structure

$$
\mathbb{J}_{4 \pi}=\left(\begin{array}{cc}
J & 4 \pi_{I}^{\sharp} \\
0 & -J^{*}
\end{array}\right)
$$

as in Theorem 2.7.

We need a few lemmas.

Lemma 4.22.

$$
L_{4 \pi}=\left\{\left(X^{0,1}+\pi^{\sharp} \xi^{1,0}, \xi^{1,0}\right) \mid \xi^{1,0} \in \Omega^{1,0}(X), X^{0,1} \in \mathfrak{X}^{0,1}(X)\right\} .
$$

Proof. It is clear that

$$
\mathbb{J}_{4 \pi}\left(X^{0,1}, 0\right)=\left(J X^{0,1}, 0\right)=-i\left(X^{0,1}, 0\right) .
$$

On the other hand, since $\pi_{I}^{\sharp}=\frac{1}{2 i}\left(\pi^{\sharp}-\bar{\pi}^{\sharp}\right)$, it follows that

$$
\pi_{I}^{\sharp} \xi^{1,0}=\frac{1}{2 i}\left(\pi^{\sharp} \xi^{1,0}\right)=-\frac{i}{2} \pi^{\sharp} \xi^{1,0} .
$$

Since $\pi_{R}^{\sharp}=J \circ \pi_{I}^{\sharp}$, we have

$$
J \circ \pi^{\sharp}=J \circ\left(\pi_{R}^{\sharp}+i \pi_{I}^{\sharp}\right)=-\pi_{I}^{\sharp}+i \pi_{R}^{\sharp}=i\left(\pi_{R}^{\sharp}+i \pi_{I}^{\sharp}\right)=i \pi^{\sharp} .
$$


It thus follows that

$$
\mathbb{J}_{4 \pi}\left(\pi^{\sharp} \xi^{1,0}, \xi^{1,0}\right)=\left(J \pi^{\sharp} \xi^{1,0}+4 \pi_{I}^{\sharp} \xi^{1,0},-J^{*} \xi^{1,0}\right)=\left(-i \pi^{\sharp} \xi^{1,0},-i \xi^{1,0}\right)=-i\left(\pi^{\sharp} \xi^{1,0}, \xi^{1,0}\right) .
$$

Hence, $\left(X^{0,1}+\pi^{\sharp} \xi^{1,0}, \xi^{1,0}\right)$ is an eigenvector of $\mathbb{J}_{4 \pi}$ with eigenvalue $-i$. The conclusion thus follows from dimension counting.

By abuse of notations, $\nabla$ denotes both the $T^{0,1} X$-representation on $\left(T^{1,0} X\right)_{\pi}^{*}$ and the $\left(T^{1,0} X\right)_{\pi}^{*}$-representation on $T^{0,1} X$.

Lemma 4.23. For any $X^{0,1} \in \mathfrak{X}^{0,1}(X)$ and $\xi^{1,0} \in \Omega^{1,0}(X)$, we have

$$
\nabla_{X^{0,1}} \xi^{1,0}=L_{X^{0,1}} \xi^{1,0}
$$

Proof. For any $Y^{1,0} \in \mathfrak{X}^{1,0}(X)$, we have

$$
\begin{aligned}
\left\langle\nabla_{X^{0,1}} \xi^{1,0}, Y^{1,0}\right\rangle & =X^{0,1}\left\langle\xi^{1,0}, Y^{1,0}\right\rangle-\left\langle\xi^{1,0}, \nabla_{X^{0,1}} Y^{1,0}\right\rangle \\
& =X^{0,1}\left\langle\xi^{1,0}, Y^{1,0}\right\rangle-\left\langle\xi^{1,0}, \operatorname{pr}^{1,0}\left[X^{0,1}, Y^{1,0}\right]\right\rangle \\
& =X^{0,1}\left\langle\xi^{1,0}, Y^{1,0}\right\rangle-\left\langle\xi^{1,0},\left[X^{0,1}, Y^{1,0}\right]\right\rangle \\
& =\left\langle L_{X^{0,1}} \xi^{1,0}, Y^{1,0}\right\rangle .
\end{aligned}
$$

Hence, $\nabla_{X^{0,1}} \xi^{1,0}=L_{X^{0,1}} \xi^{1,0}$.

Lemma 4.24. For any $X^{0,1} \in \mathfrak{X}^{0,1}(X)$ and $\xi^{1,0} \in \Omega^{1,0}(X)$, we have

$$
\pi^{\sharp} \nabla_{X^{0,1}} \xi^{1,0}=\operatorname{pr}^{1,0}\left[\pi^{\sharp} \xi^{1,0}, X^{0,1}\right] .
$$

Proof. Note that if $Y^{1,0} \in \mathfrak{X}^{1,0}(X)$ is a holomorphic vector field, then $\operatorname{pr}^{1,0}\left[X^{0,1}, Y^{1,0}\right]=0$. Hence, it follows that $L_{X^{0,1}} \pi \in \Gamma\left(T^{0,1} X \wedge T^{1,0} X\right)$, for $\pi \in \mathfrak{X}^{2,0}(X)$ is a holomorphic bivector field. Therefore, $\left(L_{X^{0,1}} \pi\right)^{\sharp} \xi^{1,0} \in \mathfrak{X}^{0,1}(X)$, that is, $\operatorname{pr}^{1,0}\left(L_{X^{0,1}} \pi\right)^{\sharp} \xi^{1,0}=0$. Now, since

$$
\pi^{\sharp}\left(L_{X^{0,1}} \xi^{1,0}\right)-L_{X^{0,1}}\left(\pi^{\sharp} \xi^{1,0}\right)=\left(L_{X^{0,1}} \pi\right)^{\sharp} \xi^{1,0},
$$


applying $\mathrm{pr}^{1,0}$ on both sides, we obtain

$$
\pi^{\sharp}\left(L_{X^{0,1}} \xi^{1,0}\right)=\operatorname{pr}^{1,0}\left[X^{0,1}, \pi^{\sharp} \xi^{1,0}\right]
$$

and, using equation (28), we have

$$
\pi^{\sharp}\left(\nabla_{X^{0,1}} \xi^{1,0}\right)=\operatorname{pr}^{1,0}\left[X^{0,1}, \pi^{\sharp} \xi^{1,0}\right] .
$$

Proof of Theorem 4.21. First, recall that the Lie bracket on $\Gamma\left(L_{4 \pi}\right)$ is the restriction of the Courant bracket

$$
\llbracket X+\xi, Y+\eta \rrbracket=[X, Y]+\mathcal{L}_{X} \eta-\mathcal{L}_{Y} \xi+\frac{1}{2} d(\xi Y-\eta X)
$$

of $\Gamma\left(T X \oplus T^{*} X\right)[17,41]$.

Consider the map

$$
\phi: T^{0,1} X \bowtie\left(T^{1,0} X\right)_{\pi}^{*} \rightarrow L_{4 \pi}:\left(X^{0,1}, \xi^{1,0}\right) \mapsto\left(X^{0,1}+\pi^{\sharp} \xi^{1,0}, \xi^{1,0}\right),
$$

which is an isomorphism of vector bundles. It is clear that $\phi$ interchanges the anchor maps. One immediately sees that

$$
\begin{aligned}
& {\left[\phi\left(X^{0,1}\right), \phi\left(Y^{0,1}\right)\right]=\phi\left[X^{0,1}, Y^{0,1}\right] \quad \forall X^{1,0}, Y^{1,0} \in \mathfrak{X}^{1,0}(X)} \\
& {\left[\phi\left(\xi^{1,0}\right), \phi\left(\eta^{1,0}\right)\right]=\phi\left[\xi^{1,0}, \eta^{1,0}\right] \quad \forall \xi^{1,0}, \eta^{1,0} \in \Omega^{1,0}(X) .}
\end{aligned}
$$

Now, for any $X^{0,1} \in \mathfrak{X}^{0,1}(X)$ and $\xi^{1,0} \in \Omega^{1,0}(X)$,

$$
\begin{array}{rlrl} 
& {\left[\phi\left(X^{0,1}\right), \phi\left(\xi^{1,0}\right)\right]} & \\
= & \llbracket X^{0,1}, \pi^{\sharp} \xi^{1,0}+\xi^{1,0} \rrbracket & & \text { (by definition of } \phi) \\
= & {\left[X^{0,1}, \pi^{\sharp} \xi^{1,0}\right]+L_{X^{0,1}} \xi^{1,0}} & & \text { (by definition of } \llbracket \cdot, \cdot \rrbracket) \\
= & {\left[X^{0,1}, \pi^{\sharp} \xi^{1,0}\right]+\nabla_{X^{0,1}} \xi^{1,0}} & & \text { (by equation (28)) } \\
= & \operatorname{pr}^{1,0}\left[X^{0,1}, \pi^{\sharp \xi^{1,0}}\right]+\operatorname{pr}^{0,1}\left[X^{0,1}, \pi^{\sharp \xi^{1,0}}\right]+\nabla_{X^{0,1}} \xi^{1,0} & & \text { (since } \left.T_{\mathbb{C}} X=T^{1,0} X \oplus T^{0,1} X\right) \\
= & \operatorname{pr}^{1,0}\left[X^{0,1}, \pi^{\sharp \xi^{1,0}}\right]-\nabla_{\xi^{1,0}} X^{0,1}+\nabla_{X^{0,1}} \xi^{1,0} & & \text { (by equation (20)). }
\end{array}
$$


On the other hand,

$$
\phi\left[X^{0,1}, \xi^{1,0}\right]=\phi\left(-\nabla_{\xi^{1,0}} X^{0,1}+\nabla_{X^{0,1}} \xi^{1,0}\right)=-\nabla_{\xi^{1,0}} X^{0,1}+\pi^{\sharp} \nabla_{X^{0,1}} \xi^{1,0}+\nabla_{X^{0,1}} \xi^{1,0} .
$$

From equation (29), it thus follows that

$$
\left[\phi\left(X^{0,1}\right), \phi\left(\xi^{1,0}\right)\right]=\phi\left[X^{0,1}, \xi^{1,0}\right]
$$

Hence, $\phi$ is indeed a Lie algebroid isomorphism.

\subsection{Holomorphic Poisson cohomology}

We define the Poisson cohomology of a holomorphic Poisson manifold $(X, \pi)$ to be the cohomology of the holomorphic Lie algebroid $\left(T^{*} X\right)_{\pi}$. Consider the matched pair $\left(T^{0,1} X,\left(T^{1,0} X\right)_{\pi}^{*}\right)$ associated to a holomorphic Poisson manifold $(X, \pi)$. With Proposition 4.5 in mind, we set $A=T^{0,1} X$ and $B=\left(T^{1,0} X\right)_{\pi}^{*}$. Then,

$$
\Gamma\left(\wedge^{k} A^{*} \otimes \wedge^{l} B^{*}\right) \simeq \Omega^{0, k}(X) \otimes_{\mathcal{C}^{\infty}(X, \mathbb{C})} \mathfrak{X}^{l, 0}(X) \simeq \Omega^{0, k}\left(X, T^{l, 0} X\right)
$$

and the commutative diagram of Proposition 4.5 becomes

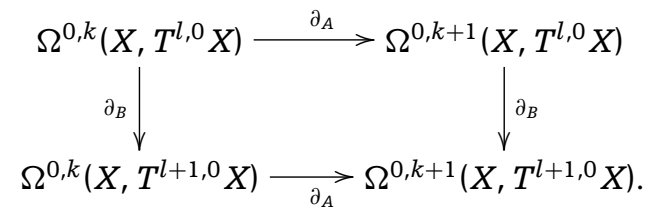

The next proposition describes the coboundary operators $\partial_{A}$ and $\partial_{B}$ in this context.

Proposition 4.25. Let $\left(T^{0,1} X,\left(T^{1,0} X\right)_{\pi}^{*}\right)$ be the matched pair associated to a holomorphic Poisson manifold $(X, \pi)$. Then,

(a) $\partial_{A}: \Omega^{0, k}\left(X, T^{l, 0} X\right) \rightarrow \Omega^{0, k+1}\left(X, T^{l, 0} X\right)$ is the $\bar{\partial}$-operator associated to the holomorphic vector bundle $T^{l, 0} X$;

(b) $\partial_{B}: \Omega^{0, k}\left(X, T^{l, 0} X\right) \rightarrow \Omega^{0, k}\left(X, T^{l+1,0} X\right)$ is the operator $d_{\pi}$ defined by the relation

$$
\left.\left(d_{\pi} \alpha\right)\left(Y_{1}, \ldots, Y_{k}\right)=\left[\pi, \alpha\left(Y_{1}, \ldots, Y_{k}\right)\right]+(-1)^{k} \alpha\right\lrcorner\left[\pi, Y_{1} \wedge \cdots \wedge Y_{k}\right]
$$


where $Y_{1}, \ldots, Y_{k}$ are arbitrary elements of $\mathfrak{X}^{0,1}(X)$ and $\alpha \downarrow\left[\pi, Y_{1} \wedge \cdots \wedge Y_{k}\right]$ denotes the element in $\mathfrak{X}^{l+1,0}(X)$, obtained by contracting $\alpha$ with the $(k+1)$ vector field $\left[\pi, Y_{1} \wedge \cdots \wedge Y_{k}\right]$.

Alternatively, if $\omega \in \Omega^{0, k}(X)$ and $P \in \mathfrak{X}^{l, 0}(X)$, then

$$
d_{\pi}(\omega \otimes P)=\omega \otimes[\pi, P]+\sum_{i=1}^{n}\left(i_{\pi^{\sharp}\left(e^{i}\right)} \mathrm{d} \omega\right) \otimes\left(e_{i} \wedge P\right),
$$

where $\left(e_{1}, \ldots, e_{n}\right)$ is a basis of $T_{X}^{1,0} X$ and $\left(e^{1}, \ldots, e^{n}\right)$ is the dual basis of $\left(T_{X}^{1,0} X\right)^{*}$.

Proof. (a) This is straightforward. (b) Since equation (31) follows easily from equation (32), we will only prove equation (32).

For any $A_{1}, \ldots, A_{k} \in \mathfrak{X}^{0,1}(X)$ and $B_{0}, \ldots, B_{l} \in \Omega^{1,0}(X)$, according to equation (19), we have

$$
\left(\partial_{B}(\omega \otimes P)\right)\left(A_{1}, \ldots, A_{k}, B_{0}, \ldots, B_{l}\right)=T \cdot \omega\left(A_{1}, \ldots, A_{k}\right)+\sum_{i=0}^{l}(-1)^{i} S_{i} \cdot P\left(B_{0}, \ldots, \widehat{B}_{i}, \ldots, B_{l}\right)
$$

Here,

$$
\begin{aligned}
T= & \sum_{j=0}^{l}(-1)^{j} \pi^{\sharp}\left(B_{j}\right) P\left(B_{0}, \ldots, \widehat{B_{j}}, \ldots, B_{l}\right) \\
& +\sum_{j_{1}, j_{2}=0}^{l}(-1)^{j_{1}+j_{2}} P\left(\left[B_{j_{1}}, B_{j_{2}}\right], B_{0}, \ldots, \widehat{B_{j_{1}}}, \ldots, \widehat{B_{j_{2}}}, \ldots, B_{l}\right)
\end{aligned}
$$

and, for $i=1, \ldots, l$,

$$
S_{i}=\pi^{\sharp}\left(B_{i}\right)\left(\omega\left(A_{1}, \ldots, A_{k}\right)\right)-\sum_{j=1}^{k} \omega\left(A_{1}, \ldots, \nabla_{B_{i}} A_{j}, \ldots, A_{k}\right) .
$$

It is clear that

$$
T=[\pi, P]\left(B_{0}, \ldots, B_{l}\right) .
$$


According to equation (20), we have $\nabla_{B_{i}} A_{j}=\operatorname{pr}^{0,1}\left[\pi^{\sharp} B_{i}, A_{j}\right]$. Since $\omega$ is a $(0, k)$-form, it follows that

$$
\left(\left[\pi^{\sharp} B_{i}, A_{j}\right]-\operatorname{pr}^{0,1}\left[\pi^{\sharp} B_{i}, A_{j}\right]\right) \downarrow \omega=0 .
$$

As a consequence, we have

$$
\omega\left(A_{1}, \ldots, \nabla_{B_{i}} A_{j}, \ldots, A_{k}\right)=\omega\left(A_{1}, \ldots,\left[\pi^{\sharp} B_{i}, A_{j}\right], \ldots, A_{k}\right) .
$$

Therefore,

$$
\begin{aligned}
S_{i} & =\pi^{\sharp}\left(B_{i}\right)\left(\omega\left(A_{1}, \ldots, A_{k}\right)\right)-\sum_{j=1}^{k} \omega\left(A_{1}, \ldots,\left[\pi^{\sharp} B_{i}, A_{j}\right], \ldots, A_{k}\right) \\
& =\left(L_{\pi^{\sharp}\left(B_{i}\right)} \omega\right)\left(A_{1}, \ldots, A_{k}\right) \\
& =\left(i_{\pi^{\sharp}\left(B_{i}\right)} \mathrm{d} \omega\right)\left(A_{1}, \ldots, A_{k}\right),
\end{aligned}
$$

where the last equality uses the relation $i_{\pi^{\sharp} B_{i}} \omega=0$. Hence, it follows that

$$
\begin{aligned}
& \sum_{i=0}^{l}(-1)^{i} S_{i} \cdot P\left(B_{0}, \ldots, \widehat{B}_{i}, \ldots, B_{l}\right) \\
& \quad=\sum_{i=0}^{l}(-1)^{i}\left(i_{\pi^{\sharp}\left(B_{i}\right)} \mathrm{d} \omega\right)\left(A_{1}, \ldots, A_{k}\right) P\left(B_{0}, \ldots, \widehat{B}_{i}, \ldots, B_{l}\right) \\
& \quad=\sum_{i=0}^{l}(-1)^{i} \sum_{j=1}^{n} B_{i}\left(e_{j}\right)\left(i_{\pi^{\sharp}\left(e^{j}\right)} \mathrm{d} \omega\right)\left(A_{1}, \ldots, A_{k}\right) P\left(B_{0}, \ldots, \widehat{B}_{i}, \ldots, B_{l}\right) \\
& =\sum_{j=1}^{n}\left(i_{\pi^{\sharp}\left(e^{j}\right)} \mathrm{d} \omega\right)\left(A_{1}, \ldots, A_{k}\right)\left(e_{j} \wedge P\right)\left(B_{0}, \ldots, B_{l}\right) .
\end{aligned}
$$

This concludes the proof of the proposition.

As an immediate consequence, we have the following corollary.

Corollary 4.26. Let $(X, \pi)$ be a holomorphic Poisson manifold. The following cohomologies are all isomorphic:

(a) the holomorphic Poisson cohomology of $(X, \pi)$;

(b) the complex Lie algebroid cohomology of $L_{4 \pi}$; 
(c) the total cohomology of the double complex

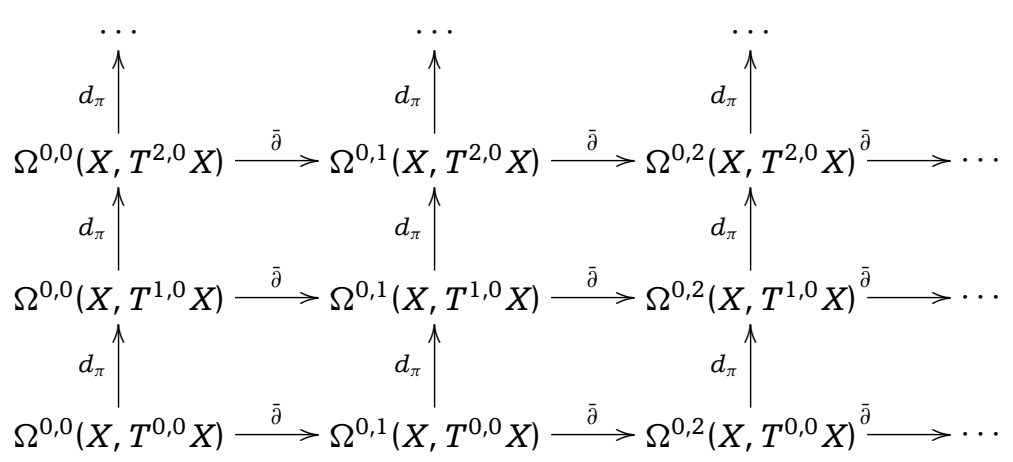

Here, $d_{\pi}$ is the differential operator defined by equation (31) or equation (32).

Remark 4.27. When $X$ is a Stein manifold (for instance, $X=\mathbb{C}^{n}$ ), one easily sees that our Poisson cohomology groups are isomorphic to the ones defined by Lichnérowicz's cochain complex of holomorphic mutivector fields, as in the smooth case (see, for instance, [38]).

We also note that our holomorphic Poisson cohomology groups are always finite dimensional if the manifold is compact.

\section{Acknowledgments}

We would like to thank Centre Émile Borel and Peking University for their hospitality while work on this project was being done. We also wish to thank Sam Evens and Alan Weinstein for useful discussions and comments. Research of M. Stiénon was supported by the European Union through the FP6 Marie Curie R.T.N. ENIGMA (Contract number MRTN-CT-2004-5652). Research of P. Xu was partially supported by the National Science Foundation (NSF) grants DMS-0306665 and DMS0605725 \& NSA grant H98230-06-1-0047.

\section{References}

[1] Block, J. "Duality and equivalence of module categories in noncommutative geometry I." (2005): preprint arXiv:math/0509284.

[2] Bondal, A. "Non-commutative deformations and Poisson brackets on projective spaces." Preprint no. 67, Max Planck Institut Bonn, 1993.

[3] Boyom, M. N. "KV-cohomology of Koszul-Vinberg algebroids and Poisson manifolds." International Journal of Mathematics 16, no. 9 (2005): 1033-61. 
[4] Brown, K. A., K. R., Goodearl, and M. Yakimov. "Poisson structures on affine spaces and flag varieties. I. Matrix affine Poisson space." Advances in Mathematics 206, no. 2 (2006): 567-629.

[5] Brylinski, J. L., and G. Zuckerman. "The outer derivation of a complex Poisson manifold." Journal für die Reine und Angewandte Mathematik 506 (1999): 181-9.

[6] Chemla, S. "A duality property for complex Lie algebroids." Mathematische Zeitschrift 232, no. 2 (1999): 367-88.

[7] Coste, A., P., Dazord, and A. Weinstein. "Groupoïdes symplectiques." Publications de l'Institut Mathématique, Nouvelle Série, vol. 87. Lyon, France: Université Claude Bernard, 1987.

[8] Crainic, M. “Generalized complex structures and Lie brackets." (2004): preprint arXiv:math/0412097.

[9] Damianou, P. A., and R. L. Fernandes. "Integrable hierarchies and the modular class." Annales de l'Institut Fourier 58, no. 1 (2008): 107-37.

[10] Drinfel'd, V. G. "Constant quasiclassical solutions of the Yang-Baxter quantum equation." Doklady Akademii Nauk SSSR. 273, no. 3 (1983): 531-5.

[11] Drinfel'd, V. G. "Hamiltonian structures on Lie groups, Lie bialgebras and the geometric meaning of classical Yang-Baxter equations." Doklady Akademii Nauk SSSR 268, no. 2 (1983): 285-7.

[12] Evens, S., and J.-H. Lu. “On the variety of Lagrangian subalgebras 1." Annales Scientifiques de l'École Normale Supérieure 34, no. 5 (2001): 631-68.

[13] Evens, S., and J.-H. Lu. "On the variety of Lagrangian subalgebras 2." Annales Scientifiques de l'École Normale Supérieure 39, no. 2 (2006): 347-79.

[14] Evens, S., and J.-H. Lu. "Poisson geometry of the Grothendieck resolution of a complex semisimple group." Moscow Mathematical Journal 7, no. 4 (2007): 613-42.

[15] Evens, S., J.-H., Lu, and A. Weinstein. "Transverse measures, the modular class and a cohomology pairing for Lie algebroids." Quarterly Journal of Mathematics 50, no. 200 (1999): 417-36.

[16] Gualtieri, M. “Branes on Poisson varieties." (2007): preprint arXiv:0710.2719.

[17] Gualtieri, M. “Generalized complex geometry." (2004): preprint arXiv:math/0401221.

[18] Hitchin, N. “Generalized Calabi-Yau manifolds." Quarterly Journal of Mathematics 54, no. 3 (2003): 281-308.

[19] Hitchin, N. "Instantons, Poisson structures and generalized Kähler geometry," Communications in Mathematical Physics 265, no. 1 (2006): 131-64.

[20] Huebschmann, J. "Poisson geometry of certain moduli spaces." The Proceedings of the Winter School Geometry and Physics (1996): 15-35.

[21] Huebschmann, J. "Duality for Lie-Rinehart algebras and the modular class." Journal für die Reine und Angewandte Mathematik 510 (1999): 103-59.

[22] Huybrechts, D. Complex Geometry: An Introduction. (Universitext). Berlin, Germany: Springer, 2005.

[23] Iwasaki, K. "Fuchsian moduli on a Riemann surface-its Poisson structure and PoincaréLefschetz duality." Pacific Journal of Mathematics 155, no. 2 (1992): 319-40. 
[24] Kapustin, A. "Topological strings on noncommutative manifolds." International Journal of Geometric Methods in Modern Physics 1, no. 1-2 (2004): 49-81.

[25] Korotkin, D., and H. Samtleben. "On the quantization of isomonodromic deformations on the torus." International Journal of Modern Physics A 12, no. 11 (1997): 2013-29.

[26] Kosmann-Schwarzbach, Y. "The Lie bialgebroid of a Poisson-Nijenhuis manifold." Letters in Mathematical Physics 38, no. 4 (1996): 421-8.

[27] Kosmann-Schwarzbach, Y., and F. Magri. "Poisson-Nijenhuis structures." Annales de l'Institut Henri Poincaré 53, no. 1 (1990): 35-81.

[28] Kosmann-Schwarzbach, Y., and F. Magri “On the modular classes of Poisson-Nijenhuis manifolds." (2006): preprint arXiv:math/0611202.

[29] Liu, Z.-J., A. Weinstein, and P. Xu. "Manin triples for Lie bialgebroids." Journal of Differential Geometry 45, no. 3 (1997): 547-74.

[30] Lu, J.-H. private communication.

[31] Lu, J.-H. "Poisson homogeneous spaces and Lie algebroids associated to Poisson actions." Duke Mathematical Journal 86, no. 2 (1997): 261-304.

[32] Mackenzie, K. C. H. "Ehresmann doubles and Drinfel'd doubles for Lie algebroids and Lie bialgebroids." (2006): preprint arXiv:math/0611799.

[33] Mackenzie, K. C. H., and P. Xu. "Lie bialgebroids and Poisson groupoids." Duke Mathematical Journal 73, no. 2 (1994): 415-52.

[34] Magri, F., and C. Morosi. "On the reduction theory of the Nijenhuis operators and its applications to Gel'fand-Dikiĭ equations." Proceedings of the IUTAM-ISIMM Symposium on Modern Developments in Analytical Mechanics, Vol. 2117 (1983): 599-626.

[35] Magri, F., and C. Morosi. “Old and new results on recursion operators: an algebraic approach to KP equation," 78-96. In Topics in Soliton Theory and Exactly Solvable Nonlinear Equations (Oberwolfach, 1986). Singapore: World Scientific, 1987.

[36] Mokri, T. "Matched pairs of Lie algebroids." Glasgow Mathematical Journal 39, no. 2 (1997): 167-81.

[37] Moraru, R. "Integrable systems associated to a Hopf surface." Canadian Journal of Mathematics 55, no. 3 (2003): 609-35.

[38] Pichereau, A. "Poisson (co)homology and isolated singularities." Journal of Algebra 299, no. 2 (2006): 747-77.

[39] Polishchuk, A. "Algebraic geometry of Poisson brackets." Journal of Mathematical Sciences 84, no. 5 (1997): 1413-44.

[40] Semenov-Tian-Shansky, M. A. "Dressing transformations and Poisson group actions." Publications of the Research Institute for Mathematical Sciences 21, no. 6 (1985): 1237-60.

[41] Stiénon, M., and P. Xu. "Poisson quasi-Nijenhuis manifolds." Communications in Mathematical Physics 270, no. 3 (2007): 709-25.

[42] Stolovitch, L. "Sur les structures de Poisson singulières." Ergodic Theory and Dynamical Systems 24, no. 5 (2004): 1833-63.

[43] Vaisman, I. “Complementary 2-forms of Poisson structures." Compositio Mathematica 101, no. 1 (1996) : 55-75. 
46 C. Laurent-Gengoux et al.

[44] Weinstein, A. "The integration problem for complex Lie algebroids," 93-109. In Geometry to Quantum Mechanics, Progress in Mathematics Series, vol. 252. Boston, MA: Birkhäuser Boston, 2007.

[45] $\mathrm{Xu}, \mathrm{P}$. "Gerstenhaber algebras and BV-algebras in Poisson geometry." Communications in Mathematical Physics 200, no. 3 (1999): 545-60. 